\title{
Kinetic study of the melanogenesis from select catecholamines in the presence of L-cysteine or other amino acids.
}

\author{
Koen P. Vercruysse ${ }^{1 *}$, Venise Govan ${ }^{1}$ and Stenesha Fortner ${ }^{1}$ \\ ${ }^{1}$ Chemistry Department, Tennessee State University, Nashville, USA \\ *Corresponding author: kvercruysse@tnstate.edu
}

\begin{abstract}
This report is a continuation of an earlier report on the effect of L-cysteine on the synthesis of melanin-like materials from catecholamines. We confirmed the pattern of results indicating that in the presence of L-cysteine the appearance of dark colors during the melanogenesis reactions is delayed, but that ultimately the presence of L-cysteine yields reaction mixtures with darker colors. The results of the current report indicate that the effect of L-cysteine is very reproducible in the cases of DOPA or dopamine, but much more variable in the cases of norepinephrine or epinephrine. The delays in color formation in the cases of norepinephrine and epinephrine were much longer compared to the cases of DOPA and dopamine. In addition to L-cysteine, we tested the effect of a few amino acids on the melanogenesis reactions. In general, we observed that the presence of the amino acids slowed, but did not delay the color formation. Given enough reaction time, the presence of amino acids did result in reaction mixtures exhibiting darker colors. In addition, some of the results obtained indicated a difference between $\alpha$-amino acids and their corresponding primary amines, e.g., tyrosine vs. tyramine.
\end{abstract}

Keywords: melanin, catecholamines, L-cysteine, amino acids

\section{Introduction}

Melanins (MNs) constitute a ubiquitous class of dark-colored pigments which can be found in all kingdoms of life. It is a heterogeneous class of pigments as a wide variety of molecules can serve as precursor for these pigments and many reviews regarding their biosynthesis, chemistry, classification and functions have been written. ${ }^{1-4}$ Despite many decades of intense research and computer modelling, there is no consensus on the precise chemical structure of these biomolecules or a precise explanation for their dark colors. ${ }^{5-6}$ The dark colors of MNs, varying from light brown to intense black, is exhibited by their broad-range, monotonic absorbance profile over the entire ultraviolet and visible region of the electromagnetic spectrum. The main MN pigments found in mammalian cells are eumelanin and pheomelanin. Eumelanin is built from L-DOPA, an oxidation product of L-tyrosine, and has a dark brown to black color. ${ }^{7}$ Pheomelanin is built from a combination of L-DOPA and L-cysteine and is typically reddish in color. $^{3,8}$

Given the extraordinary significance of color in biology ${ }^{9-10}$, any factor that can influence the type of color or the intensity of color portrayed is of importance. In addition to the more "traditional" functions attributed to pigmentation in biology, Michaelian et al. have provided arguments that "the driving force behind the origin and evolution of life has been the thermodynamic imperative of increasing the entropy production of the biosphere 
through increasing the global solar photon dissipation rate". ${ }^{11-12}$ This argument led to the perspective "that the origin of life was not a scenario of organic material organization driven by natural selection leading to "better adapted" organisms or to greater chemically stability (e.g., UV resistant organisms) but rather a scenario of the dissipative structuring of material under the imposed UV solar photon potential leading to an organization of material in space and time such that this provided more efficient routes to the dissipation of this imposed photon potential. Through similar dissipative synthesis of photochemical catalysts and cofactors, more complex biosynthetic pathways were thermodynamically selected that could produce and maintain novel pigments for dissipating not only the fundamental UVC and UVB regions but eventually the entire short wavelength region of the solar photon spectrum up to the red-edge ( $700 \mathrm{~nm}) . "{ }^{\prime 12}$ In this context, and considering the broad-range, monotonic absorbance profile exhibited by MNs, an argument can be made that MNs, so ubiquitous in nature, would constitute an evolutionary endpoint of pigment formation; best adapted to dissipate a broad range of the solar spectrum.

The case of pheomelanin is a prime and well-studied example of how the presence of an amino acid, L-cysteine in this case, can alter the physic-chemical properties of MN. The reddish color of pheomelanin has been attributed to the incorporation of L-cysteine into the MN material generated during the oxidation of L-DOPA. We have reported on the effects of the presence of L-cysteine in the auto-oxidation reactions of catecholamines. ${ }^{13}$ In stark contrast to the expected reddish color, we observed that the presence of L-cysteine consistently resulted in the generation of MN-like materials with a much darker (mostly black) appearance compared to the auto-oxidation reactions in the absence of any L-cysteine. In addition, we observed that the presence of L-cysteine consistently delayed the onset of color formation and this effect appeared to be dependent on the concentration of L-cysteine present. In plant biochemistry, the impact of amino acids on the oxidation chemistry of catecholic compounds is more established, e.g., the interaction between chlorogenic acid and amino acids. ${ }^{14-15}$ In addition to these extensive reports, there are anecdotal reports of the effect of amino acids on the oxidation chemistry of catechols, e.g., the formation of a temporary, pink-reddish color during the oxidation of catechol in the presence of proline or hydroxyl-proline. ${ }^{16^{*}}$ We observed a temporary greenish color in the auto-oxidation of pyrogallol in the presence of L-cysteine. ${ }^{\dagger}$

Thus, building on our earlier work ${ }^{13}$ we expanded our studies on the effect of L-cysteine on the melanogenesis from the four catecholamines, DOPA, dopamine, norepinephrine and epinephrine (see Figure 1). In addition, we began preliminary explorations on the effects of other amino acids on these reactions.

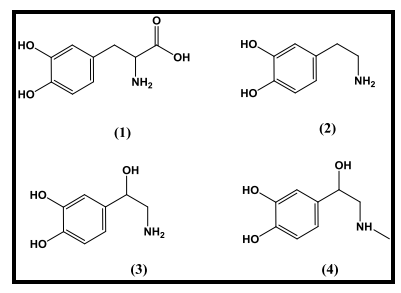

Figure 1: Chemical structures of DOPA (1), dopamine (2), norepinephrine (3) and epinephrine (4).

\footnotetext{
${ }^{*}$ Confirmed through observations in our laboratory

${ }^{\dagger}$ Observed in our laboratory
} 


\section{Materials and Methods}

\subsection{Materials and solutions}

Dopamine. $\mathrm{HCl}$ and L-cysteine. $\mathrm{HCl}$ were obtained from Alfa Aesar (Tewksbury, MA, USA). Epinephrine.HCl, norepinephrine.HCl, DOPA and all other amino acids used were obtained from Sigma-Aldrich (St Louis, MO, USA). All other chemicals used were of analytical grade. Catecholamine stock solutions and dilutions were prepared in $100 \mathrm{mM} \mathrm{Na}$-acetate buffer $(\mathrm{pH}=6.5)$. L-cysteine and other amino acid stock solutions and dilutions were prepared in $50 \mathrm{mM} \mathrm{Na}_{2} \mathrm{CO}_{3}$ solution.

\subsection{Kinetic and other experiments}

Mixtures containing $0.25 \mathrm{mM}$ catechol and various concentrations of L-cysteine or other amino acids were kept in wells of a microplate and the absorbance at $250,280,315,350$ and $400 \mathrm{~nm}$ was monitored for three hours at $37^{\circ} \mathrm{C}$. At the end of this three hour reaction time, a full UV-Vis spectrum (between 230 and 900nm) was recorded. Separately, mixtures containing $0.25 \mathrm{mM}$ catechol and L-cysteine or other amino acids at a fixed concentration were kept in test tubes in a water bath at $37^{\circ} \mathrm{C}$. Aliquots from these mixtures were diluted with SEC solvent at the start of the reaction and at various time intervals. The diluted aliquots were analyzed using SEC.

\subsection{UV-Vis spectroscopy}

UV-Vis spectroscopic measurements were made in wells of a 96-well microplate using the SynergyHT microplate reader from Biotek (Winooski, VT). For measurements involving absorbance readings below 350nm, UVtransparent microplates were used.

\subsection{Size exclusion chromatography (SEC)}

SEC analyses were performed on a Breeze 2 HPLC system equipped with two 1500 series HPLC pumps and a model 2998 Photodiode array detector from Waters, Co (Milford, MA). Analyses were performed using an Ultrahydrogel 500 column (300 X $7.8 \mathrm{~mm}$ ) obtained from Waters, Co (Milford, MA) in isocratic fashion using a mixture of $25 \mathrm{mM}$ Na-acetate:methanol:acetic acid (90:10:0.05\% v/v) as solvent. The $\mathrm{pH}$ of this solvent was measured to be 5.3 . 


\section{Results}

\subsection{UV-Vis kinetic experiments involving L-cysteine.}

Figure 2, panels A through D, illustrate some of the results obtained for the triplicate kinetic experiments involving DOPA.

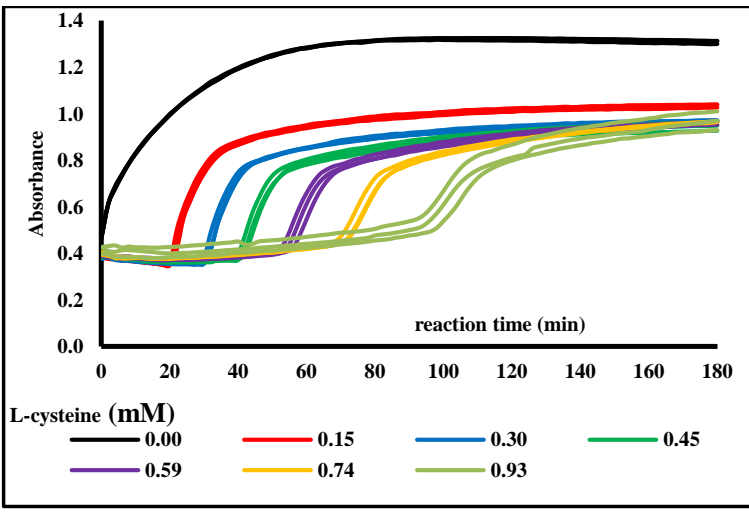

Panel A

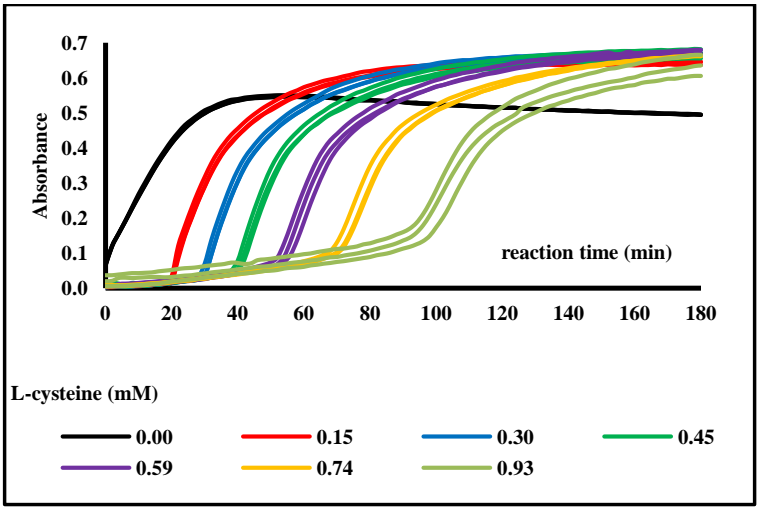

Panel C

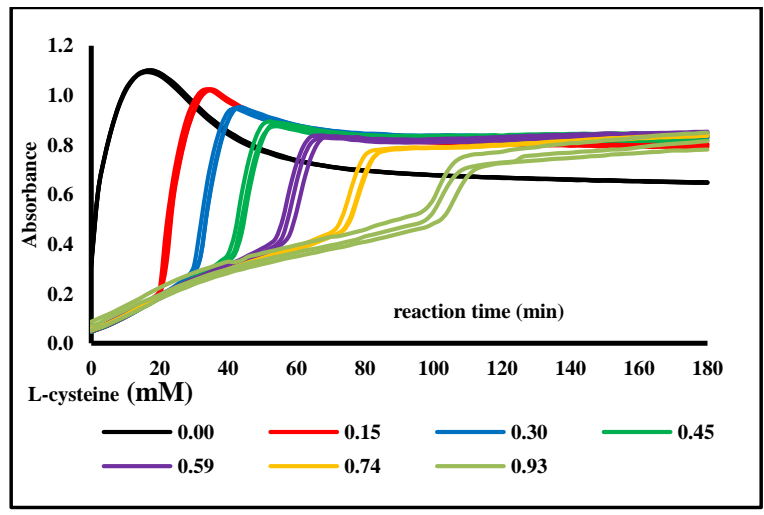

Panel B

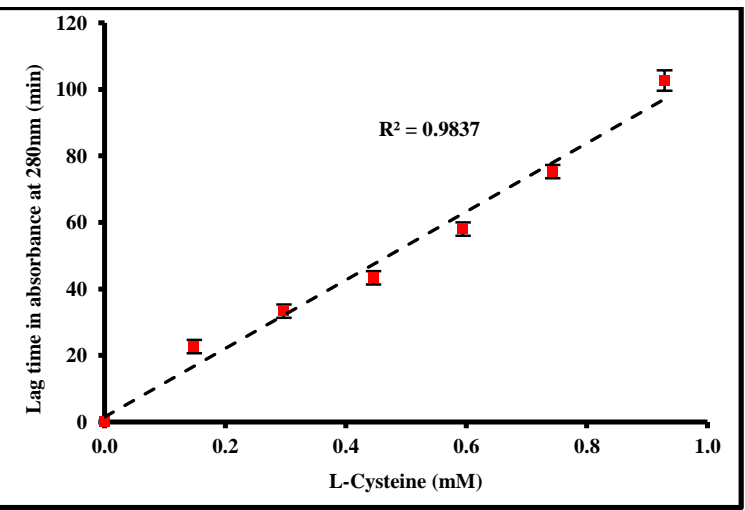

Panel D

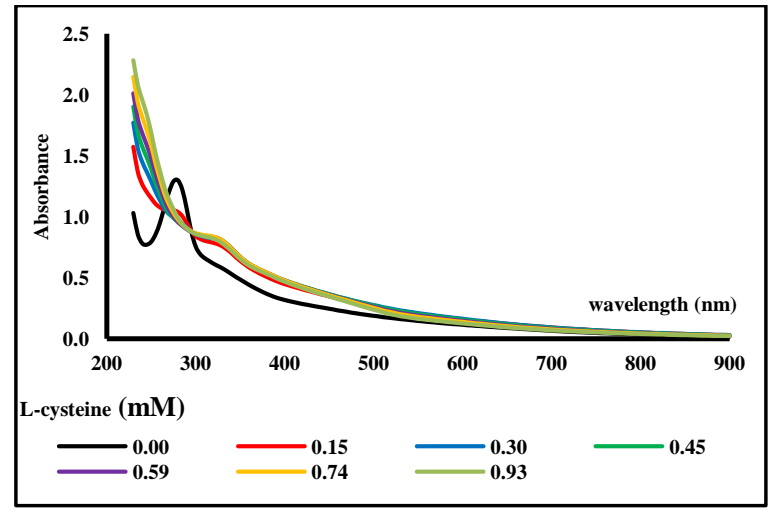

Panel E

Figure 2: Results of the kinetic experiments of the auto-oxidation of DOPA $(0.25 \mathrm{mM})$ in the presence of L-cysteine (between 0 and $0.93 \mathrm{mM})$. The panels illustrate the change in absorbance at $280 \mathrm{~nm}$ (panel A), $315 \mathrm{~nm}$ (panel B) or $350 \mathrm{~nm}$ (panel C) as a function of reaction time; the correlation between the L-cysteine concentration and the lag time between the start of the reaction and the observed increase in absorbance at 280nm (panel D); and the UV-Vis spectra of the reaction mixtures after three hours of reaction. All experiments were performed in triplicate and the individual results obtained are shown in panels A through $\mathrm{C}$, however, panel $\mathrm{E}$ shows only one set of data as a representation of the overall results. 
A number of observations can be made from the kinetic profiles and results shown in Figure 2:

1) For all experiments performed, the absorbance changed in a reproducible manner as the reactions progressed.

2) In the presence of L-cysteine, there was a significant delay or lag time between the start of the reaction and the observed changes in absorbance. The length of this lag time was independent of the wavelength monitored, but was dependent on the concentration of L-cysteine present. The relationship between the concentration of L-cysteine and this lag time is shown in panel $\mathrm{D}$ for the absorbance measurements at $280 \mathrm{~nm}$.

3) The changes in absorbance at $280 \mathrm{~nm}$ shown in panel A indicate a fast increase followed by a plateau. Despite the fact that DOPA has a strong absorbance band at $280 \mathrm{~nm}$, no decline in absorbance at $280 \mathrm{~nm}$ is to be observed. This is probably due to two facts. First, the MN-like material typically generated through the auto-oxidation of DOPA exhibits absorbance at $280 \mathrm{~nm}$. In addition, as discussed in an earlier report ${ }^{13}$, the auto-oxidation of catecholamines results in materials that appear to contain significant amounts of unoxidized precursor units. Thus the changes in the absorbance pattern at $280 \mathrm{~nm}$ is probably due to a combination of the disappearance of DOPA balanced by the appearance of MN-like material and the presence of unoxidized DOPA units exhibiting the strong absorbance band around $280 \mathrm{~nm}$.

4) The UV-Vis spectrum shown in panel $E$ of the reaction mixture without any L-cysteine shows a very strong absorbance band around 280nm (also weakly present in the reaction mixture containing the lowest concentration of L-cysteine). Previous studies and the current work performed in our laboratory indicated that, in the absence of any L-cysteine and under the reaction conditions used in the current experiments, all of the DOPA can be expected to have reacted away after three hours. Thus, the strong absorbance band at $280 \mathrm{~nm}$ present in the spectrum of the reaction mixture without L-cysteine can be assumed to reflect the presence of unoxidized DOPA units.

5) Any distinct absorbance band around $280 \mathrm{~nm}$ was absent in the spectra of the reactions containing the higher concentrations of L-cysteine. Instead, the spectra of these reaction mixtures contained an absorbance shoulder around 320nm. This absorbance shoulder may well reflect the presence of unreacted intermediates (DOPA-based or cysteine-based adducts) embedded in the final material. The rise and fall in the absorbance at $315 \mathrm{~nm}$ shown in panel B may reflect the appearance and disappearance of reaction intermediates as it is well established that the oxidation of DOPA involves various intermediates with limited lifetimes. ${ }^{17-19}$

6) The kinetic profiles for the absorbance at $350 \mathrm{~nm}$ shown in panel C illustrate the steady increase and subsequent plateau in the absorbance at that wavelength. In the presence of L-cysteine, independent of its concentration, the absorbance at the end of the reaction appeared to be about $40 \%$ higher compared to the absorbance for the reaction in the absence of any L-cysteine. This pattern of results was observed for all wavelengths between 350 and $800 \mathrm{~nm}$ (results not shown) and is a reflection of the fact 
that, in the presence of L-cysteine, the color of the endproduct is darker compared to the color of the endproduct of the material generated in the absence of any L-cysteine.

Figure 3, panels A through E, illustrate some of the results obtained for the kinetic experiments involving dopamine.

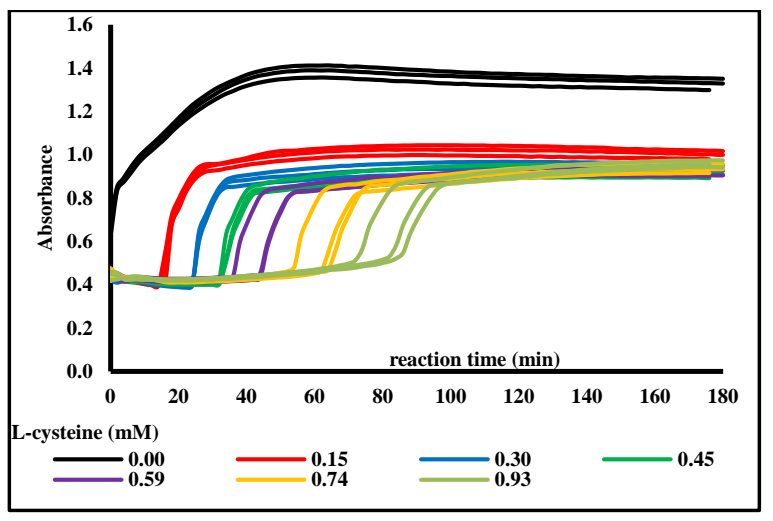

Panel A

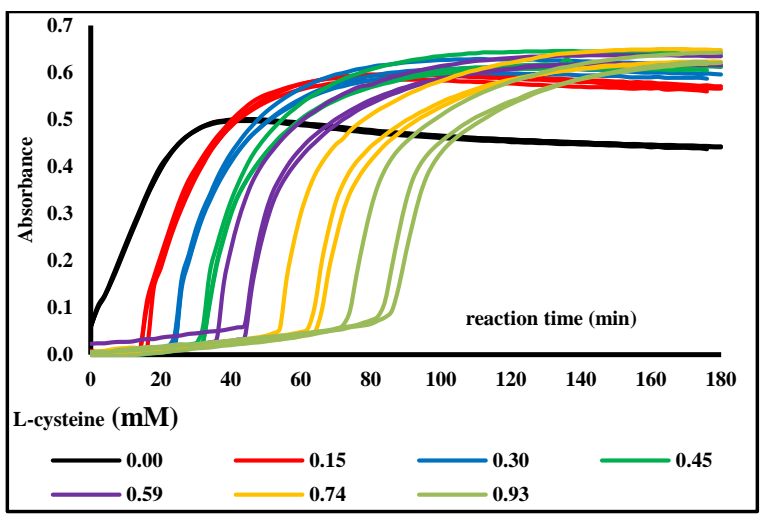

Panel C

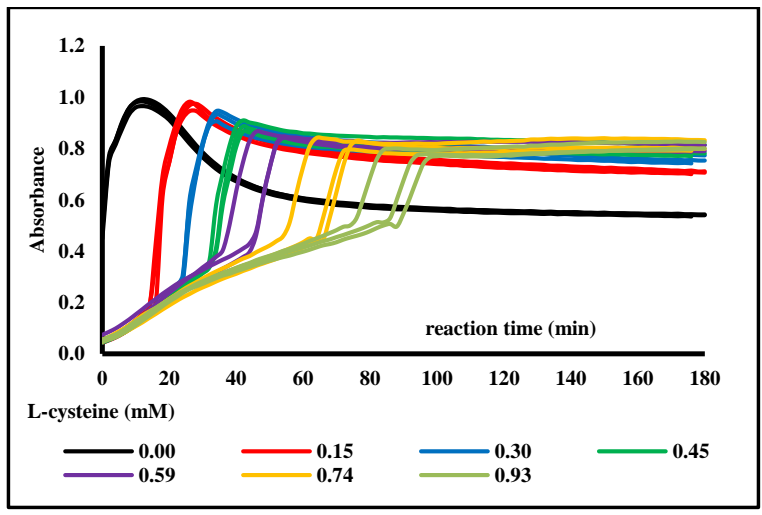

Panel B

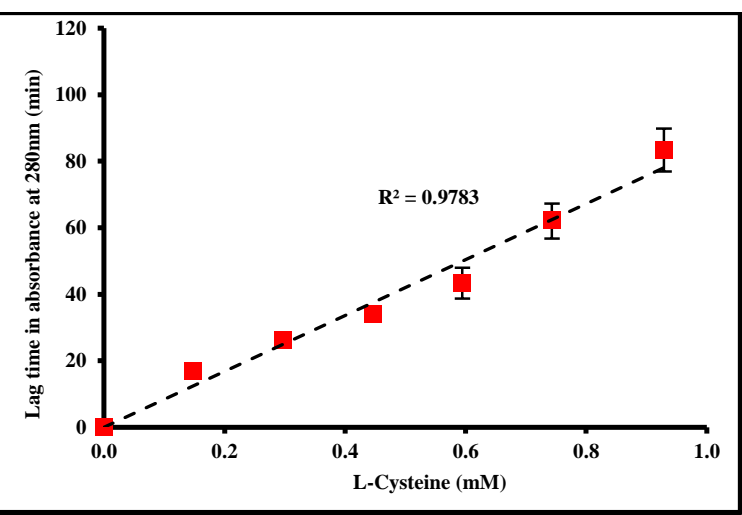

Panel D

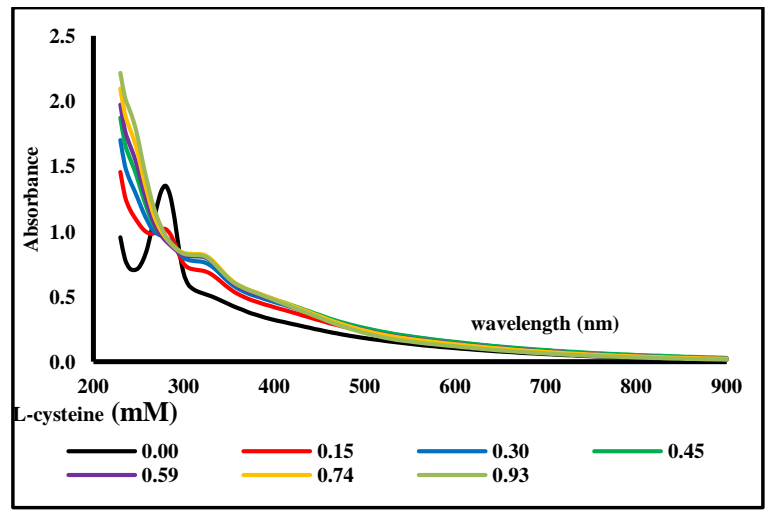

Panel E

Figure 3: Results of the kinetic experiments of the auto-oxidation of dopamine $(0.25 \mathrm{mM})$ in the presence of L-cysteine (between 0 and $0.93 \mathrm{mM})$. The panels illustrate the change in absorbance at $280 \mathrm{~nm}$ (panel A), $315 \mathrm{~nm}$ (panel B) or $350 \mathrm{~nm}$ (panel C) as a function of reaction time; the correlation between the L-cysteine concentration and the lag time between the start of the reaction and the observed increase in absorbance at 280nm (panel D); and the UV-Vis spectra of the reaction mixtures after three hours of reaction. All experiments were performed in triplicate and the individual results obtained are shown in panels A through $\mathrm{C}$, however, panel $\mathrm{E}$ shows only one set of data as a representation of the overall results. 
The pattern of results obtained for dopamine as presented in Figure 3, panels A through E, is very similar to the pattern of results obtained for DOPA. Thus, the discussions of the results obtained for DOPA can be applied to the results obtained for dopamine.

Figure 4, panels A through E, illustrate some of the results obtained for the kinetic experiments involving norepinephrine.

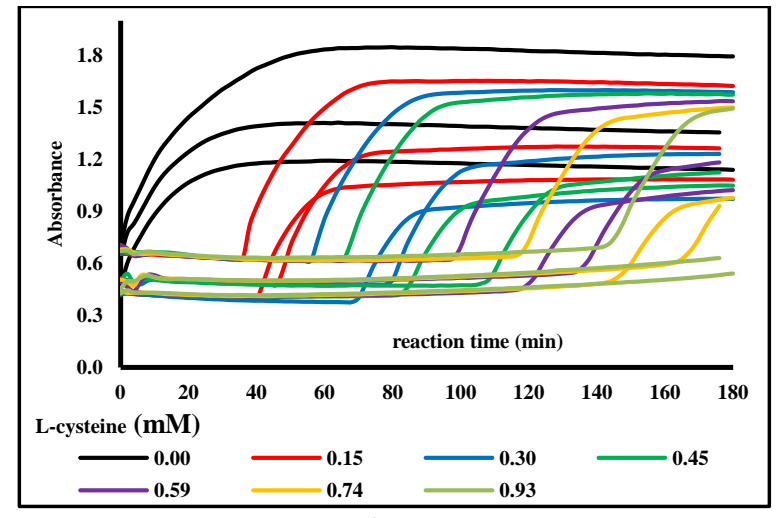

Panel A

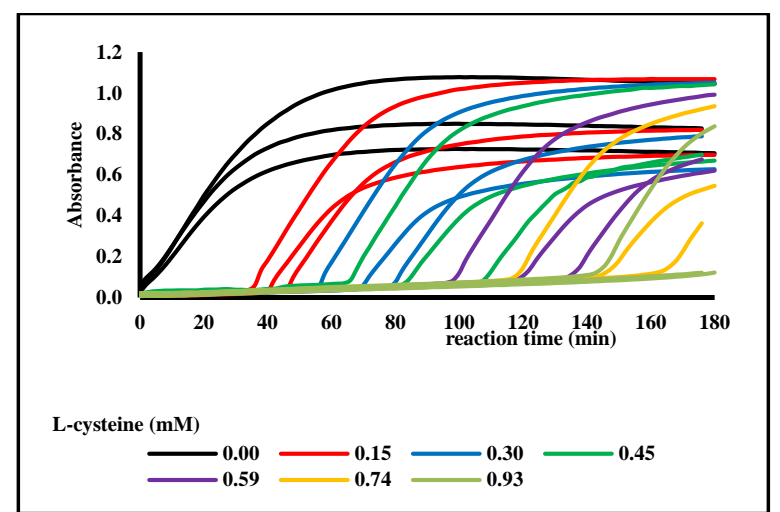

Panel C

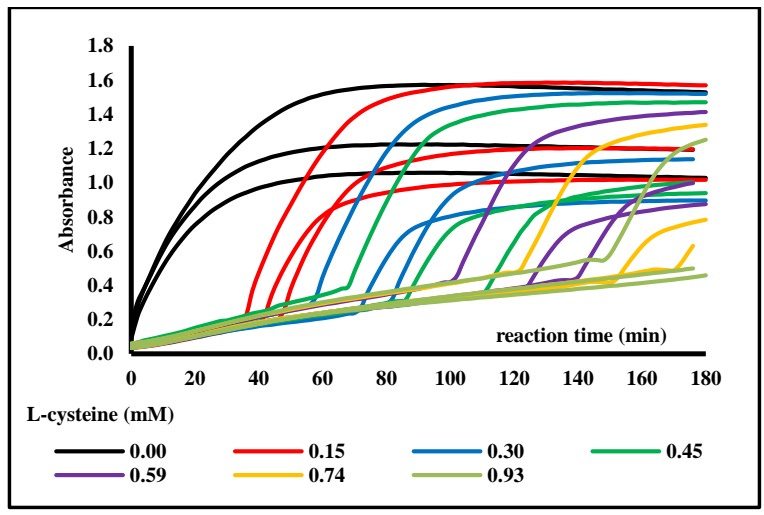

Panel B

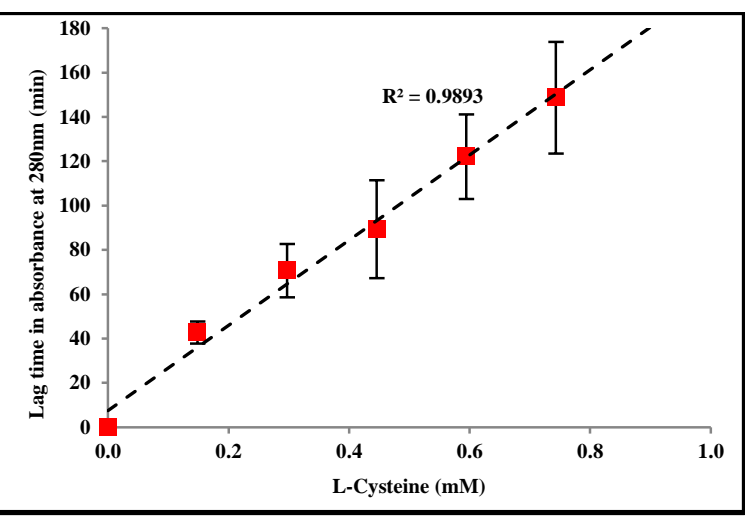

Panel D

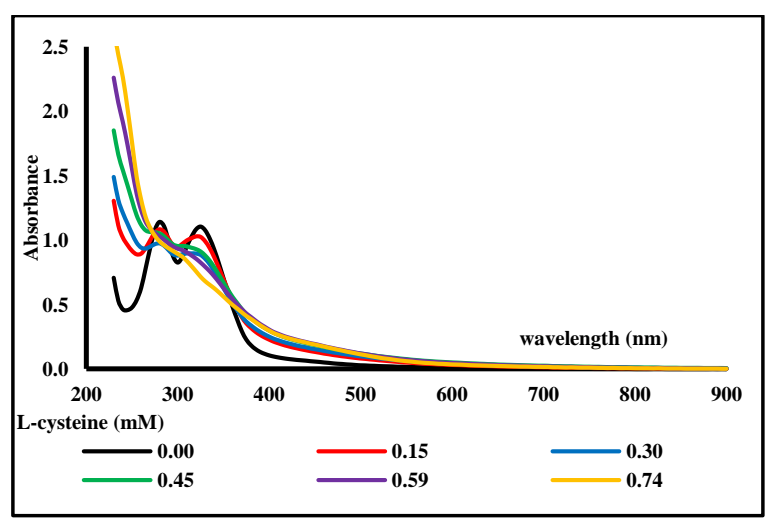

Panel E

Figure 4: Results of the kinetic experiments of the auto-oxidation of norepinephrine $(0.25 \mathrm{mM})$ in the presence of L-cysteine (between 0 and $0.93 \mathrm{mM}$ ). The panels illustrate the change in absorbance at $280 \mathrm{~nm}$ (panel A), $315 \mathrm{~nm}$ (panel B) or $350 \mathrm{~nm}$ (panel C) as a function of reaction time; the correlation between the L-cysteine concentration and the lag time between the start of the reaction and the observed increase in absorbance at 280nm (panel D); and the UV-Vis spectra of the reaction mixtures after three hours of reaction. All experiments were performed in triplicate and the individual results obtained are shown in panels A through $\mathrm{C}$, however, panel $\mathrm{E}$ shows only one set of data as a representation of the overall results. 
A number of observations can be made from the kinetic profiles and results shown in Figure 4.

1) Overall, the pattern of results obtained for the case of norepinephrine indicate that, just as for the cases of DOPA and dopamine, the presence of L-cysteine delayed the onset of absorbance increase at all wavelengths studied. However, the kinetic profiles obtained were much less reproducible compared to the cases of DOPA or dopamine. In addition, the lag times between the start of the reaction and the observed increase in absorbance in the case of norepinephrine appeared to be significantly longer compared to the lag times observed for the cases of DOPA and dopamine. This to the point that in the case of the highest concentration of L-cysteine tested $(0.93 \mathrm{mM})$, no increase in absorbance was observed within the three hours of reaction time that was monitored.

2) After three hours of reaction, the UV-Vis spectrum of the reaction mixture without any L-cysteine shows two distinct absorbance bands: one at $280 \mathrm{~nm}$ and a second at $325 \mathrm{~nm}$. These distinct absorbance bands can also clearly be observed in the UV-Vis spectra of the reactions at the lower $(0.15$ and $0.30 \mathrm{mM}$ ) concentrations of L-cysteine, but are much less distinguishable for the reactions in the presence of the higher concentrations of L-cysteine. The absorbance band at $280 \mathrm{~nm}$ can be attributed to the presence of unoxidized norepinephrine units, while the absorbance band at $325 \mathrm{~nm}$ could be attributed to the presence of an intermediate that does not react away or only very slowly. The absence of these strong absorbance bands in the reaction mixtures containing the higher concentrations of Lcysteine may indicate the different reaction paths these mixtures take due to the presence of Lcysteine. $^{20}$

3) Despite the possibility of slower reaction kinetics and different reaction pathways, the results shown in panel E indicate that in the presence of L-cysteine the absorbance of the reaction mixtures in the visible range is consistently higher compared to the reaction mixture without any L-cysteine. This is reflected in the fact that in the presence of L-cysteine, the reaction mixtures consistently have a much darker appearance compared to the mixture without any L-cysteine.

Figure 5, panels A through E, illustrate some of the results obtained for the kinetic experiments involving epinephrine.

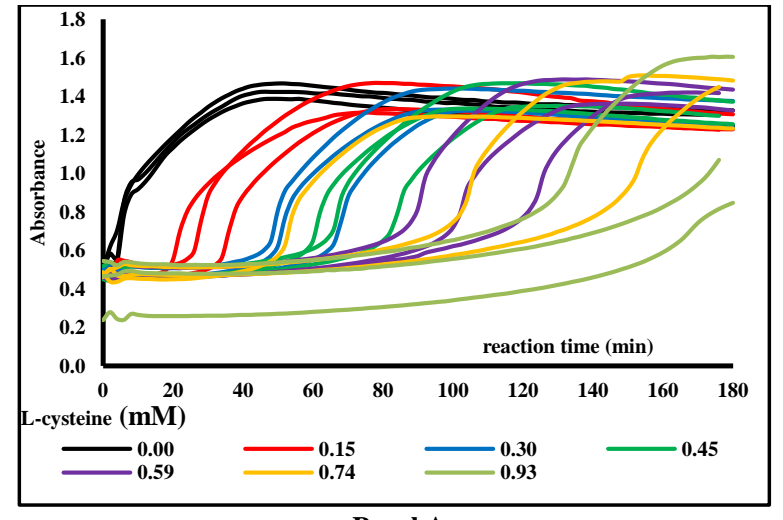

Panel A

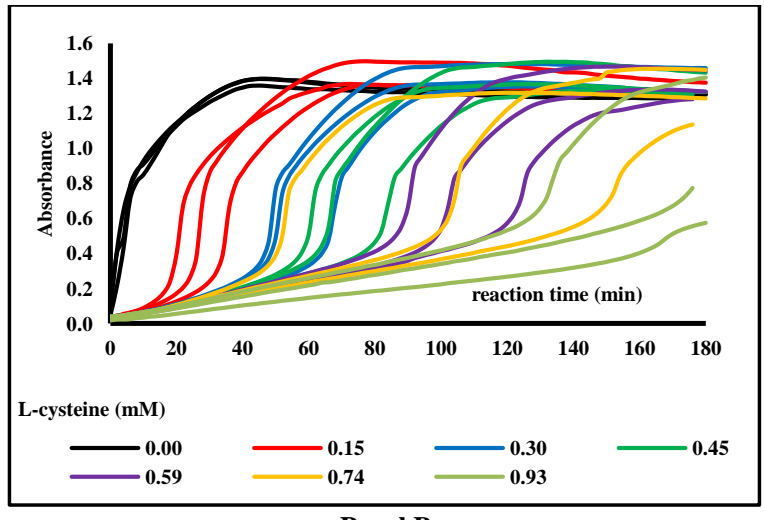

Panel B 


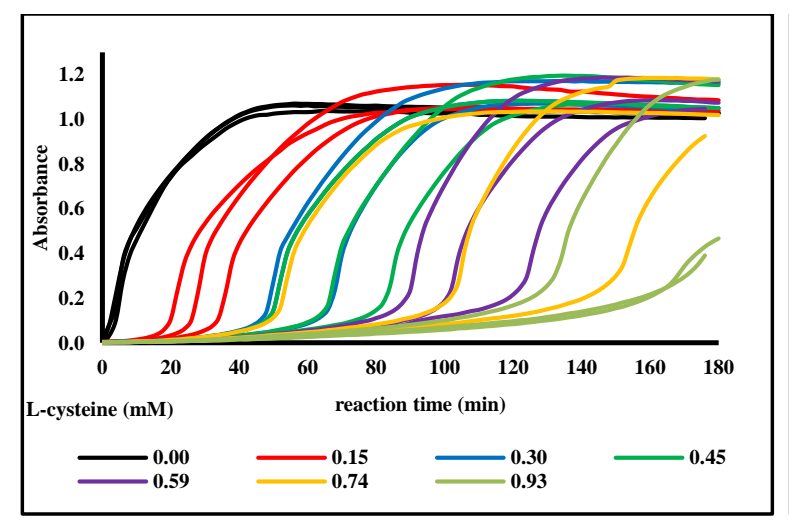

Panel C

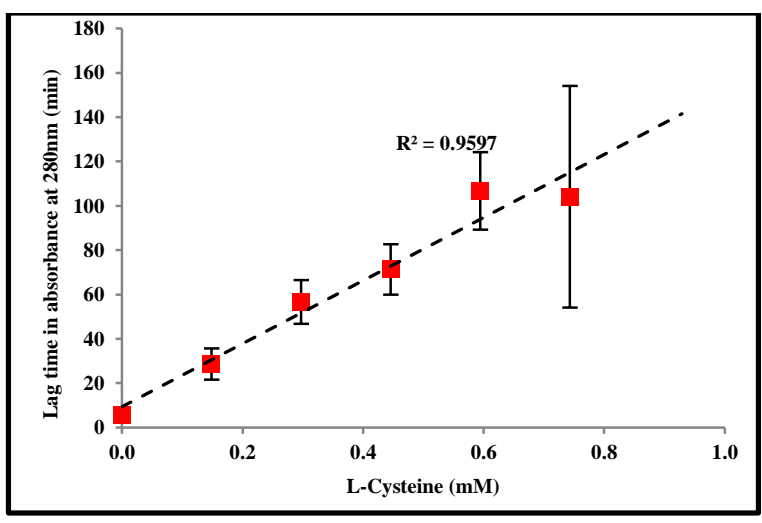

Panel D

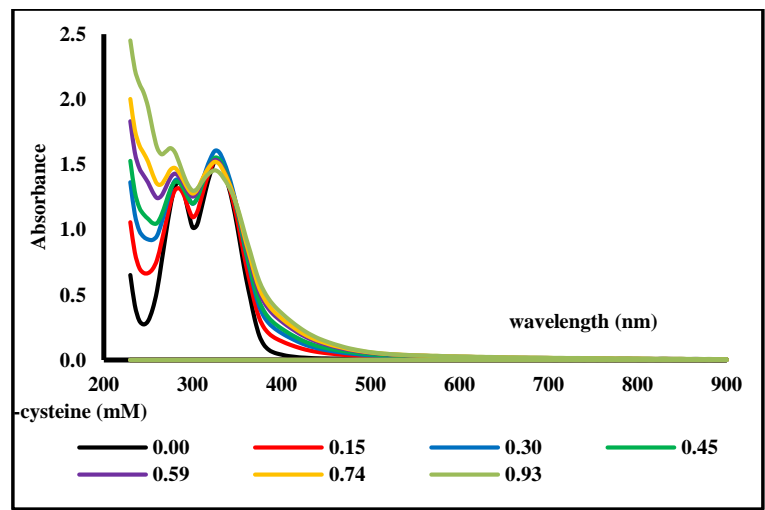

Panel E

Figure 5: Results of the kinetic experiments of the auto-oxidation of epinephrine $(0.25 \mathrm{mM})$ in the presence of L-cysteine (between 0 and $0.93 \mathrm{mM}$ ). The panels illustrate the change in absorbance at $280 \mathrm{~nm}$ (panel A), $315 \mathrm{~nm}$ (panel B) or $350 \mathrm{~nm}$ (panel C) as a function of reaction time; the correlation between the L-cysteine concentration and the lag time between the start of the reaction and the observed increase in absorbance at 280nm (panel D); and the UV-Vis spectra of the reaction mixtures after three hours of reaction. All experiments were performed in triplicate and the individual results obtained are shown in panels A through $\mathrm{C}$, however, panel E shows only one set of data as a representation of the overall results.

Overall, the pattern of results observed for the case of epinephrine is very similar to the pattern of results obtained for the case of norepinephrine. Thus, the discussions of the results obtained for norepinephrine can be applied to the results obtained for epinephrine.

An overall comparison of the pattern of results shown in Figures 2 through 4 and from our earlier report ${ }^{13}$, indicate that DOPA and dopamine behave similarly, while norepinephrine and epinephrine behave similarly, but differently from the first two. The presence of the aliphatic alcohol group within the structures of norepinephrine and epinephrine (see Figure 1) appears to set those two catecholamines apart when it comes to the generation of MN-like materials. 


\subsection{UV-Vis kinetic experiments involving other amino acids.}

In addition to our studies involving the effects of L-cysteine, we studied (in single experiments) the effects of other amino acids on the kinetics of the auto-oxidation of dopamine. It is important to point out that the concentrations at which these other amino acids were tested are much higher than the concentrations of L-cysteine used in the experiments described above. The results obtained for these experiments are shown in Figure 6, panels A through R.

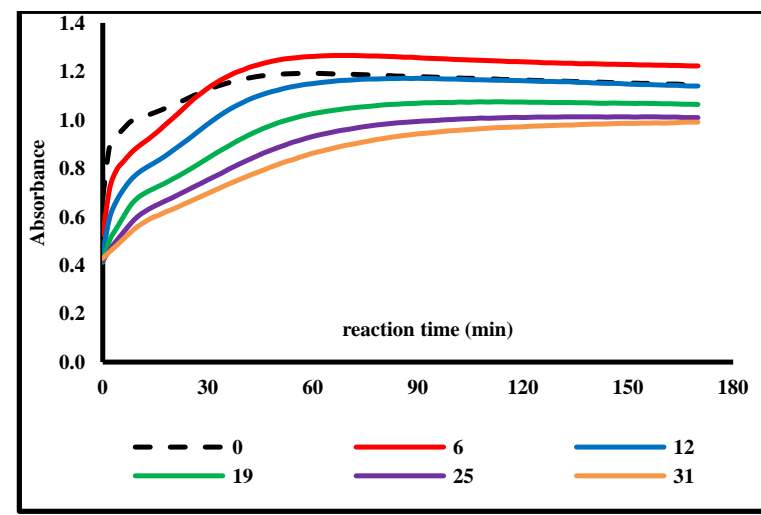

Panel A

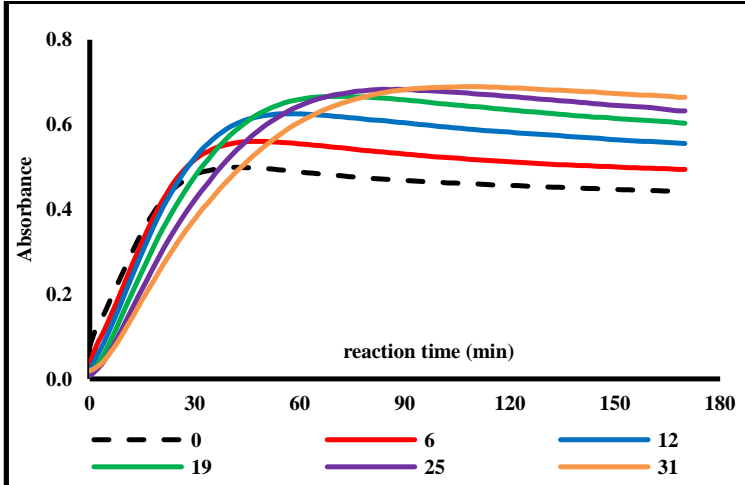

Panel C

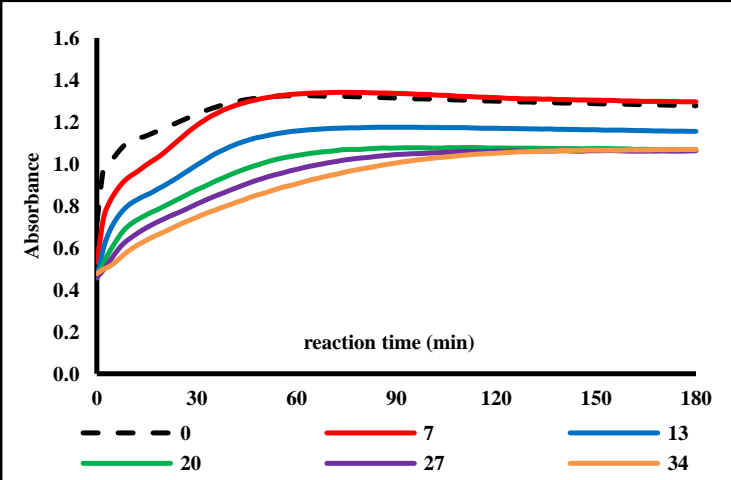

Panel E

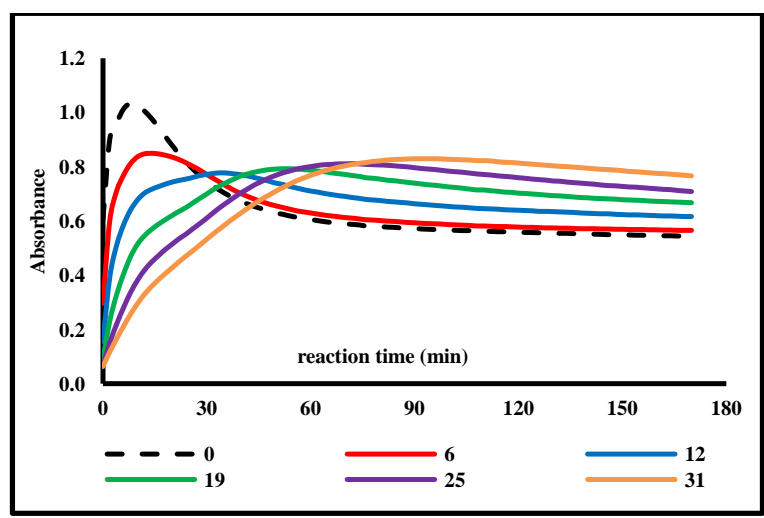

Panel B

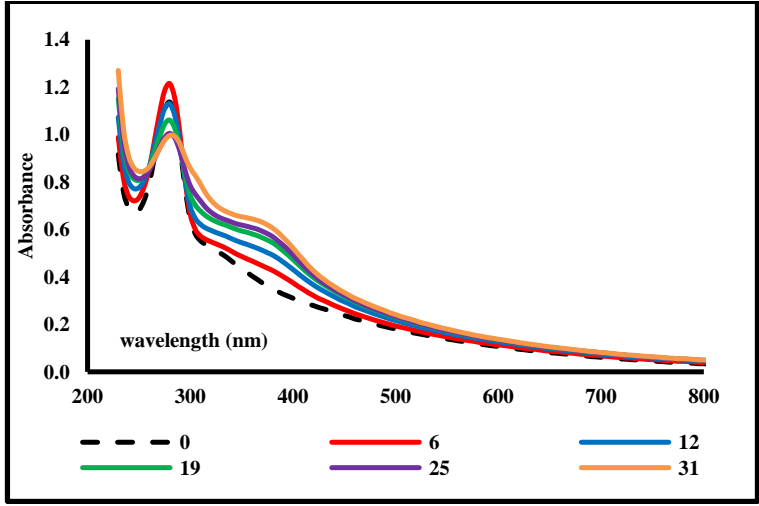

Panel D

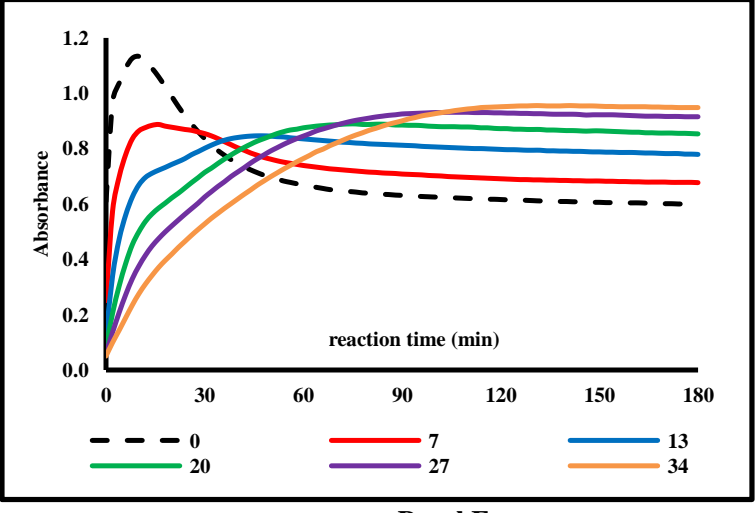

Panel F 


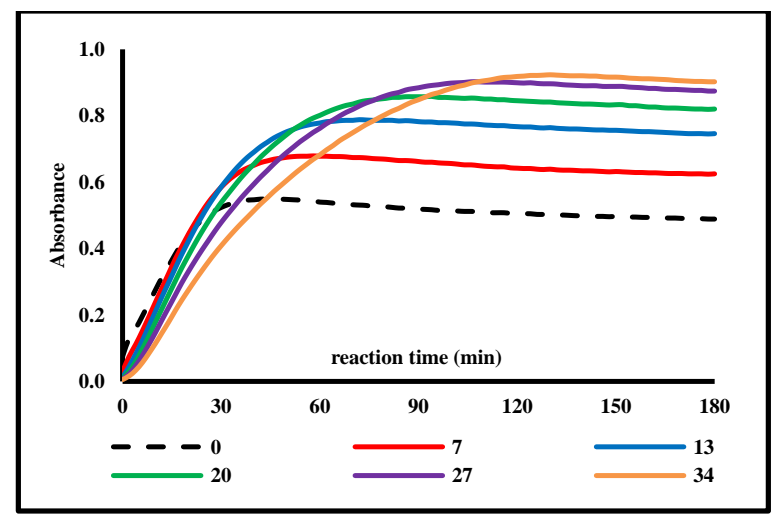

Panel G

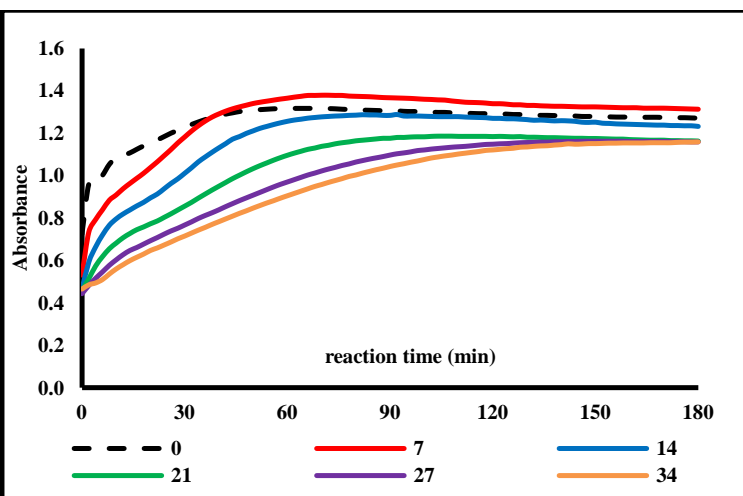

Panel I

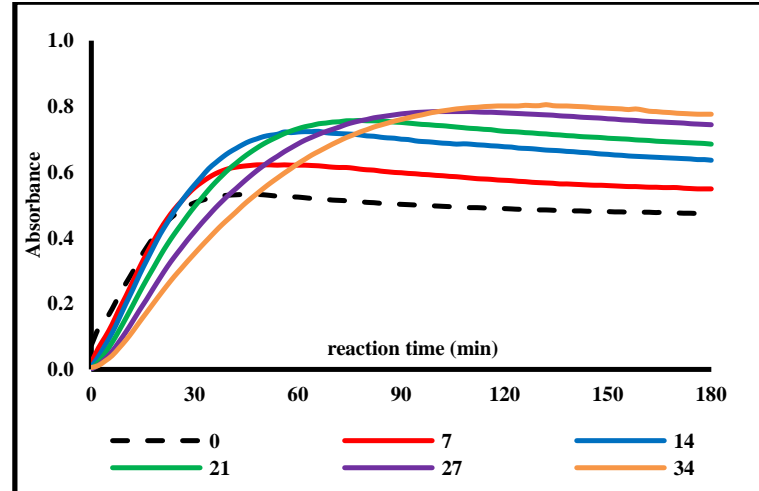

Panel K

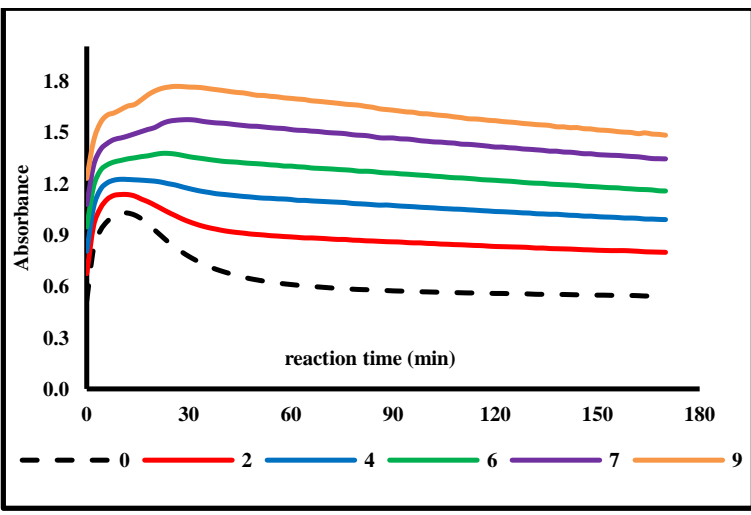

Panel M

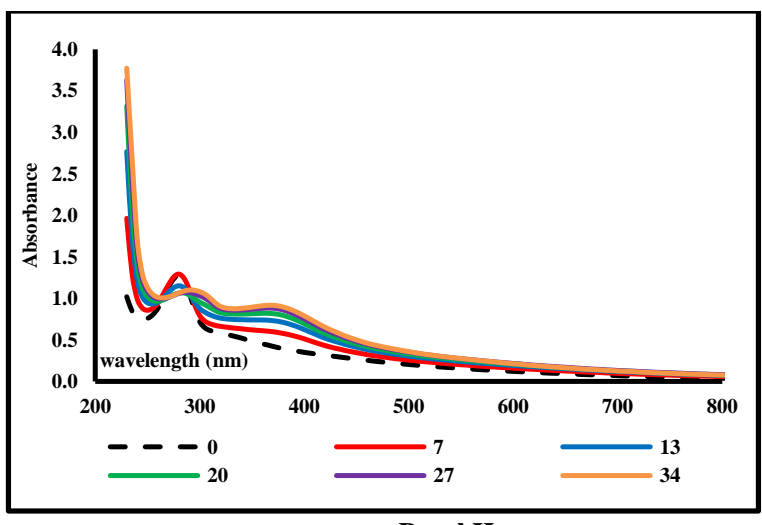

Panel H

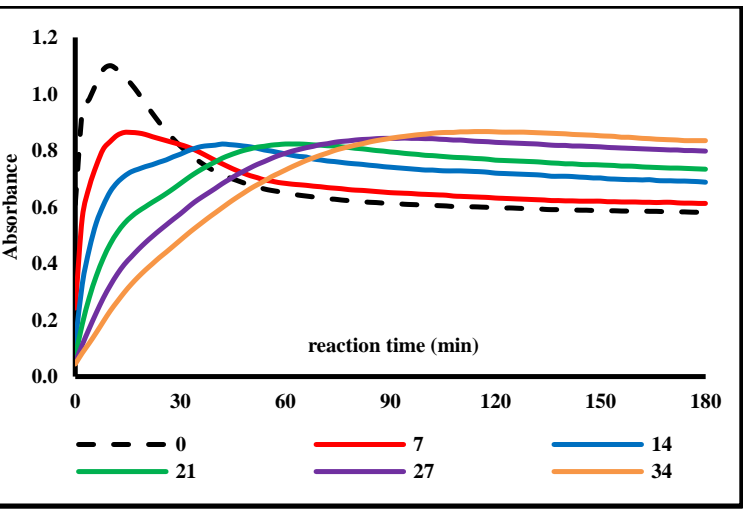

Panel J

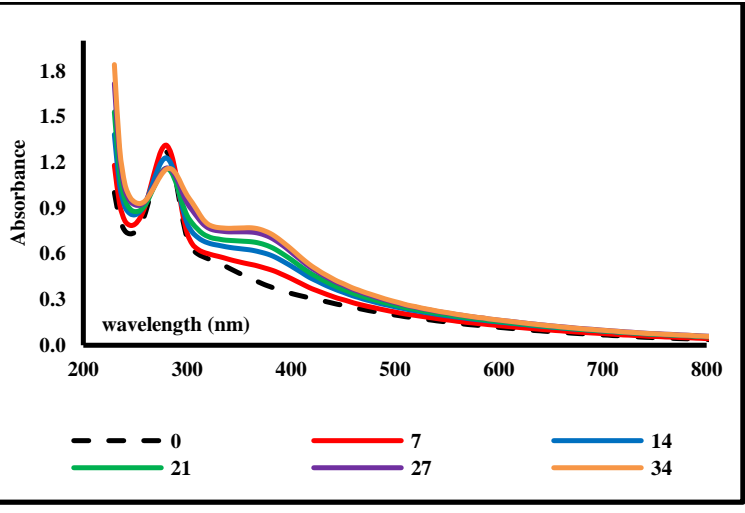

Panel L

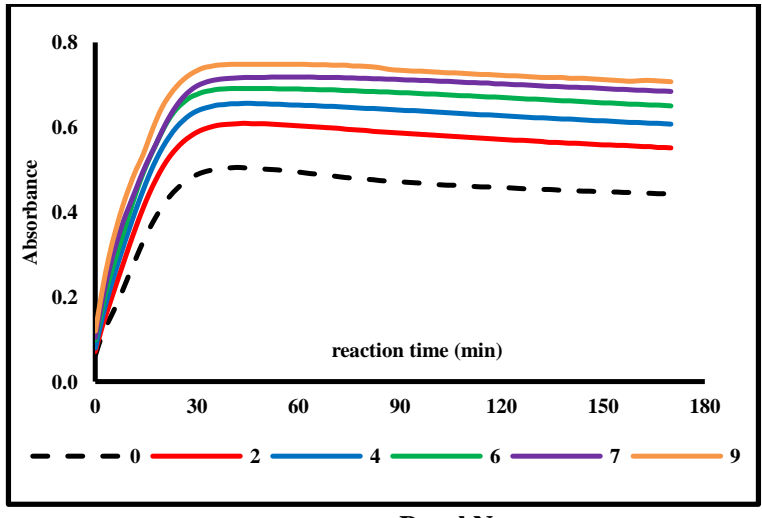

Panel N 


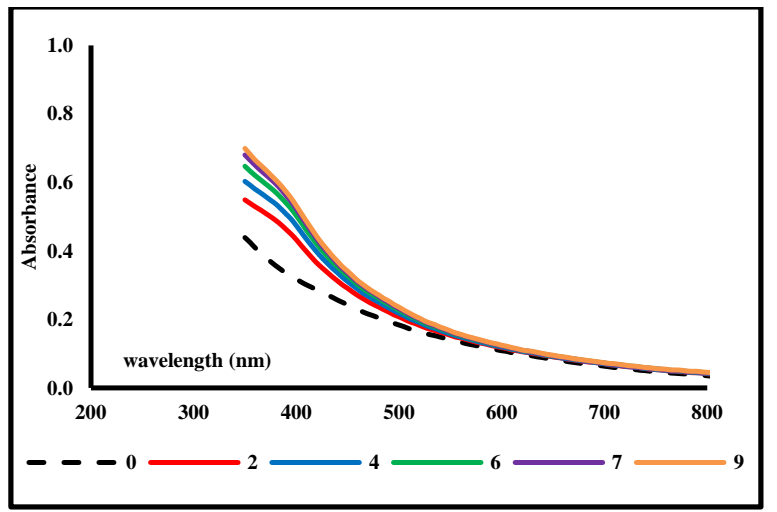

Panel O

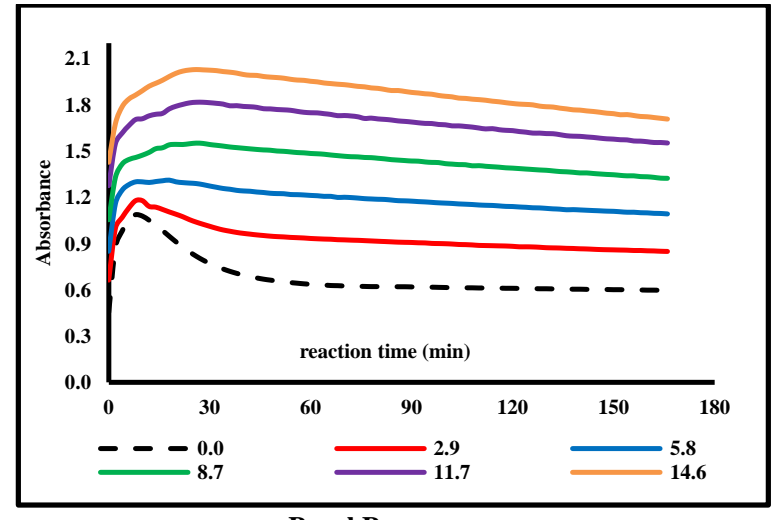

Panel P

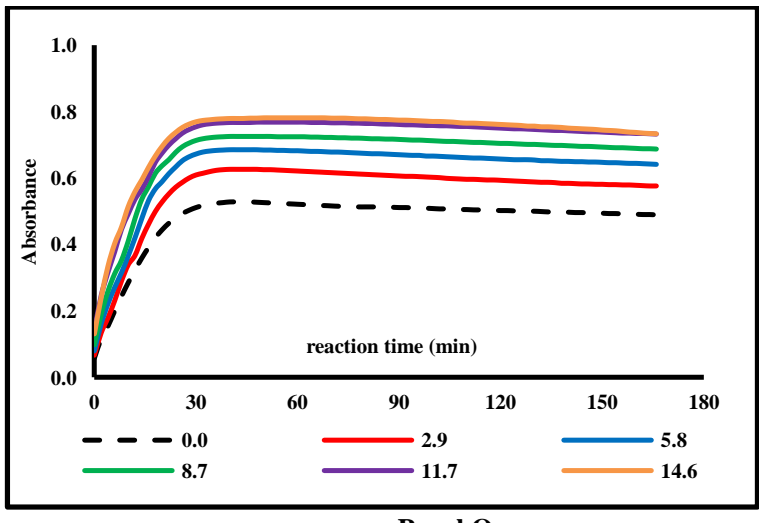

Panel Q

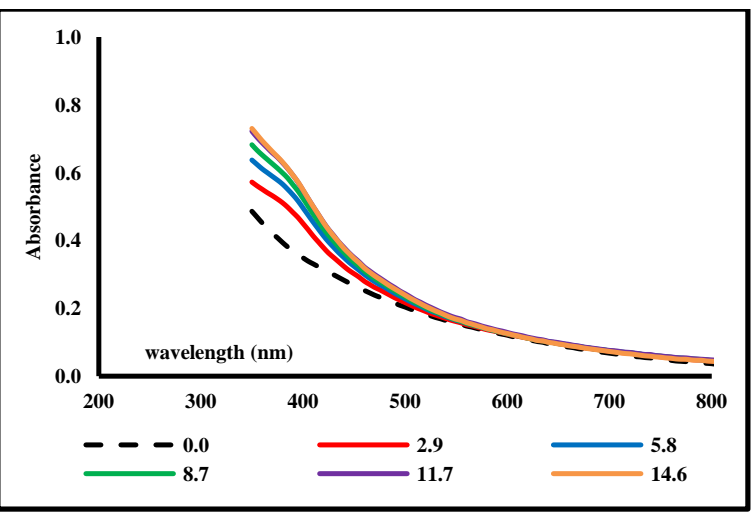

Panel R

Figure 6: Results of the kinetic experiments of the auto-oxidation of dopamine $(0.25 \mathrm{mM})$ in the presence of varying concentrations of serine (panels A through D), methionine (panels E through $\mathrm{H}$ ), glutamine (panels I through L), tyrosine (panels $\mathrm{M}$ through $\mathrm{O}$ ) or tyramine (panels $\mathrm{P}$ through R). The panels illustrate the change in absorbance at 280nm (panels A, E and I), 315nm (panels B, F, J, M, and P) or 350nm (panels C, $\mathrm{G}, \mathrm{K}, \mathrm{N}$ and $\mathrm{Q}$ ) as a function of reaction time, and the UV-Vis spectra of the reaction mixtures after three hours of reaction (panels D, H, L, O and R).

In Figure 6, the kinetic profiles at 280nm for the experiments involving tyrosine or tyramine are not shown as the absorbance readings at that wavelength were dominated by the absorbance bands at $280 \mathrm{~nm}$ exhibited by both tyrosine and tyramine. For the same reason, the UV-Vis spectra (panels $\mathrm{O}$ and $\mathrm{R}$ in Figure 6) obtained for the reaction mixtures involving tyrosine and tyramine were cut off at $350 \mathrm{~nm}$. 
In general, the following observations can be made:

1) When looking over the initial phases (first hour) of the various kinetic profiles shown in Figure 6, one can observe a pattern showing that with increasing concentrations of amino acid, the increase in absorbance is slower, but does not get delayed as is for the case of L-cysteine.

2) For the reactions performed in the presence of serine, methionine or glutamine, the UV-Vis spectra taken after three hours of reaction (panels D, H and L) show a strong absorbance band around 280nm.

However, the absorbance at that wavelength appeared to decrease with increasing concentration of amino acid.

3) For all the amino acids tested, a broad absorbance shoulder between 360 and 380nm appears in the UVVis spectra of the reaction mixtures. The intensity of this absorbance shoulder appears to increase with increasing concentration of amino acid present.

4) The case of methionine is unique amongst the amino acids tested, as the UV-Vis spectra of the reaction mixtures containing the higher concentrations of this amino acid, show a distinct absorbance band around $300 \mathrm{~nm}$ that is not present in the absence of any amino acid or in the presence of the other amino acids that were tested (see panel $\mathrm{H}$ ).

In addition to the observations discussed above, the absorbance over almost the entire visible region of the electromagnetic spectrum was enhanced with increasing concentrations of the amino acids tested; particularly in the case of methionine. These results are illustrated in Figure 7, panels A through C.

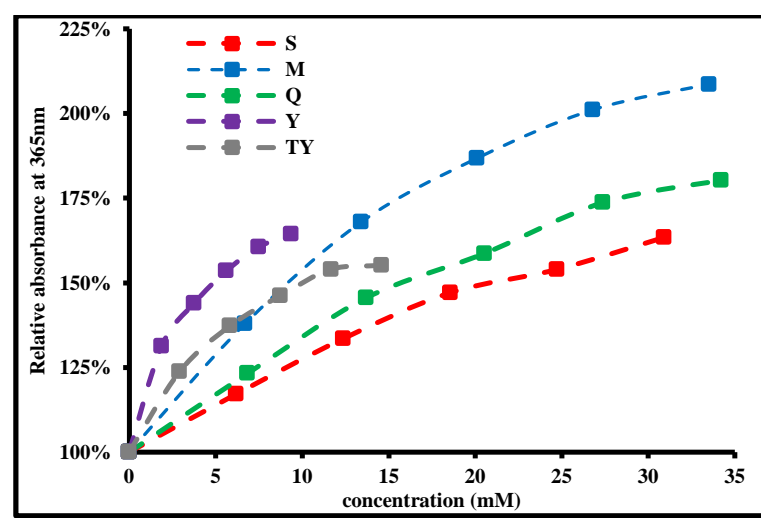

Panel A

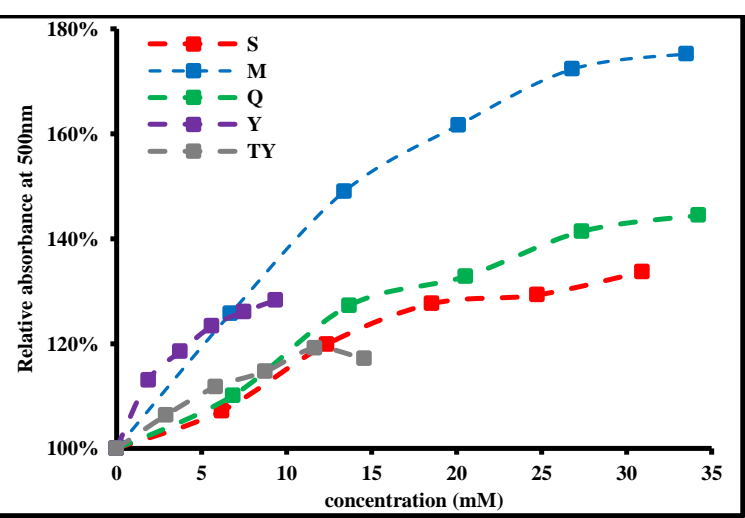

Panel B 


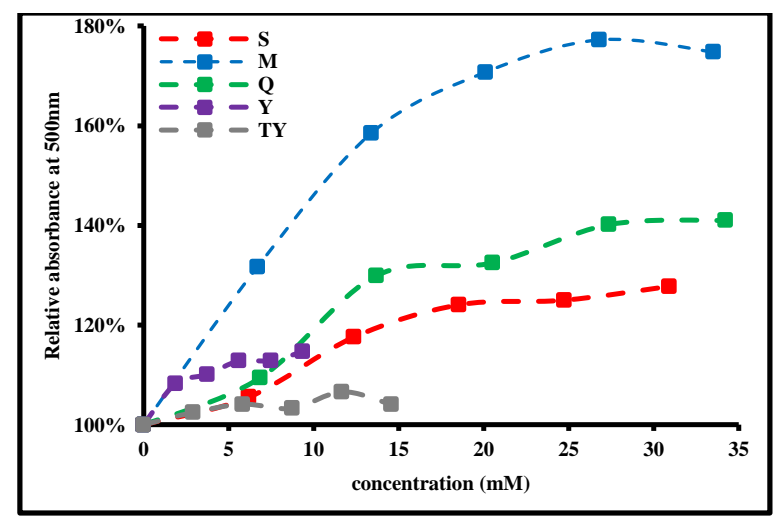

Panel C

Figure 7: Relative absorbance at 365nm (panel A), 500nm (panel B) and 600nm (panel C) as a function of concentration of serine (S), methionine (M), glutamine (Q), tyrosine (Y) or tyramine (TY) based upon the UV-Vis spectra shown in Figure 6, panels D, H, L, O and R.

The results presented in Figure 7 do suggest that the presence of amino acids affects the UV-Vis spectrum of the auto-oxidation products obtained from dopamine. The results shown in Figure 7, panels B and C, on the relative increases in absorbance at 500 or $600 \mathrm{~nm}$ are indicative of the appearance of a darker color when dopamine is oxidized in the presence of amino acids compared to its auto-oxidation in the absence of any amino acids. It is interesting to note that tyramine, as the only primary amine tested that is not an $\alpha$-amino acid, appeared to have the least effect on the apparent color of the reaction mixture.

\subsection{SEC studies involving dopamine and L-cysteine or other amino acids}

Reaction mixtures similar to the ones employed in the UV-Vis kinetic studies were set up and sampled for SEC analyses. Figure 8, panels A and B, illustrate some of the results obtained for reactions involving dopamine by itself.

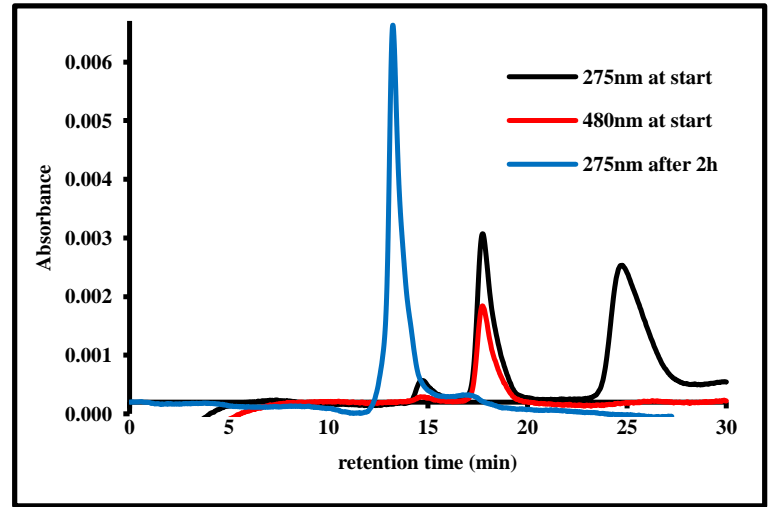

Panel A

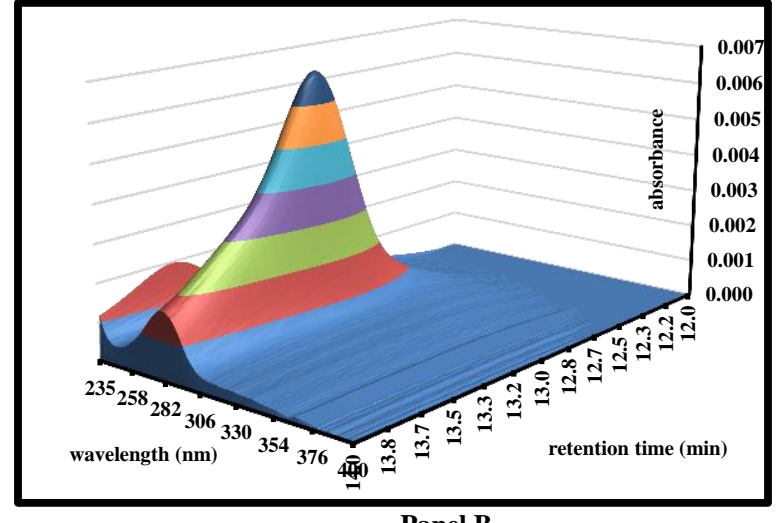

Panel B

Figure 8: SEC profiles at $275 \mathrm{~nm}$ or $480 \mathrm{~nm}$ for a reaction mixture containing $0.25 \mathrm{mM}$ dopamine kept at $37^{\circ} \mathrm{C}$ and the $\mathrm{UV}-\mathrm{Vis}$ spectra between 235 and $400 \mathrm{~nm}$ of the signal recorded between 12 and 14 minutes retention time (panel B).

In the absence of any amino acids, the auto-oxidation of dopamine proceeds rapidly and, under the conditions employed, almost all of the dopamine originally present reacted away within 30 minutes after the start of the reaction. Upon mixing dopamine, dissolved in acetate buffer, with $\mathrm{Na}_{2} \mathrm{CO}_{3}$ solution, the color of the mixture instantly changes form colorless to orange-yellow. Within 30 minutes, the reaction mixture had turned brown. Even 
when the reaction mixture is sampled immediately upon mixing the solutions, any oxidation chemistry had occurred already. Thus, the profiles "at start" shown in Figure 8, panel A, reflect the status of the reaction mixture a few seconds after the actual start of the reaction. When viewed at $275 \mathrm{~nm}$ (see Figure 8, panel A), the SEC profile shows a peak around 25 minutes that corresponds to unreacted dopamine, a peak around 17.2 minutes with strong absorbance at 300 (not shown) and 480nm and a peak around 15 minutes that shows absorbance around 300nm (results not shown). The peaks with retention times of 15 and 17.2 minutes could still be observed after about 30 minutes of reaction, but were absent after about 60 minutes of reaction. After 60 minutes of reaction, the SEC profiles of these reaction mixtures are dominated by the peak with a retention time of around 13.2 minutes and with a UV-Vis absorbance profile dominated by a strong absorbance around 280nm as shown in Figure 8, panel B. Based upon the literature ${ }^{17-19}$ it is reasonable to assume that the peaks observed around 15 and 17.2 minutes represent some of the intermediates, e.g., dihydroxyindole (DHI), that are known to be generated in the initial phases of the melanogenesis reactions.

Figure 9, panels A and B, illustrate some of the SEC results obtained for a similar reaction mixture as for Figure 8, but containing $0.6 \mathrm{mM}$ L-cysteine. The mixture was colorless at the start of the reaction; turning light yellow after about 30 minutes of reaction; turning dark yellow after about $1.5 \mathrm{~h}$ of reaction; turning brownish after about $2.5 \mathrm{~h}$ of reaction. About $80 \%$ of the dopamine had reacted away after about 30 minutes of reaction and almost all of the dopamine had reacted away after about 90 minutes of reaction.

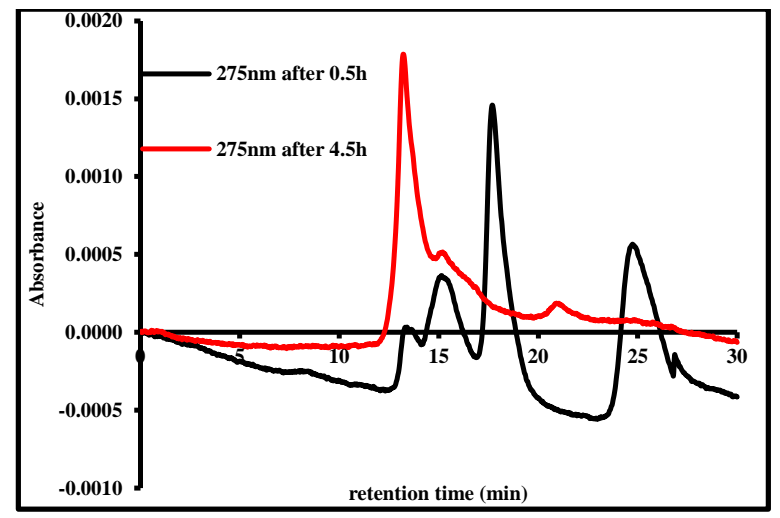

Panel A

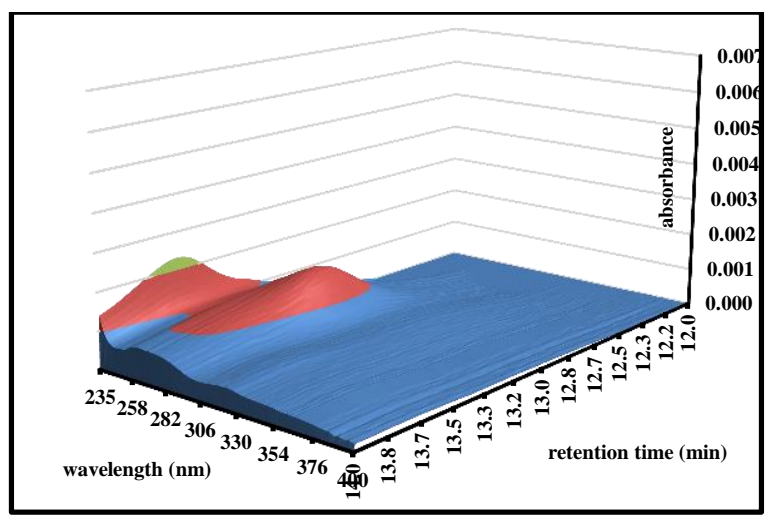

Panel B

Figure 9: SEC profiles at $275 \mathrm{~nm}$ (after 0.5 and $4.5 \mathrm{~h}$ reaction) for a reaction mixture containing $0.25 \mathrm{mM}$ dopamine and $0.6 \mathrm{mM}$ L-cysteine kept at $37^{\circ} \mathrm{C}$ and the UV-Vis spectra between 235 and $400 \mathrm{~nm}$ of the signal recorded between 12 and 14 minutes retention time (panel B; shown at the same scale as for Figure 8, panel B) for the reaction mixture after 4.5h of reaction.

The results shown in Figure 9, panel A, indicate that, in the presence of L-cysteine, the same profile of peaks was obtained after about $0.5 \mathrm{~h}$ of reaction as for the profile of peaks obtained at the start of the reaction in the absence of any dopamine (see Figure 8, panel A). After $4.5 \mathrm{~h}$ of reaction, all the dopamine had reacted away and the SEC profile is dominated by a peak with retention time of about 13.2 minutes, but peaks of intermediates or other compounds can be observed around 15 and 21 minutes of retention times. The peak with retention time of 21 minutes was not observed at the start of this reaction, nor was it observed for the reaction mixture containing dopamine without any L-cysteine (see Figure 8, panel A). The UV-Vis spectrum of the peak around 13.2 minutes does not exhibit the 
dominating absorbance band around $280 \mathrm{~nm}$. An observation that would be in line with the results obtained for the kinetic experiments discussed above (see section 3.1).

Figure 10, panels A and B, illustrate some of the SEC results obtained for a similar reaction mixture as for Figure 8 , but containing $0.6 \mathrm{mM}$ serine. The mixture was light yellow at the start of the reaction, turning light brown after about 30 minutes of reaction; turning brown after $1 \mathrm{~h}$ of reaction. About $90 \%$ of the dopamine had reacted away after about 90 minutes of reaction and almost all of the dopamine had reacted away after about 120 minutes of reaction.

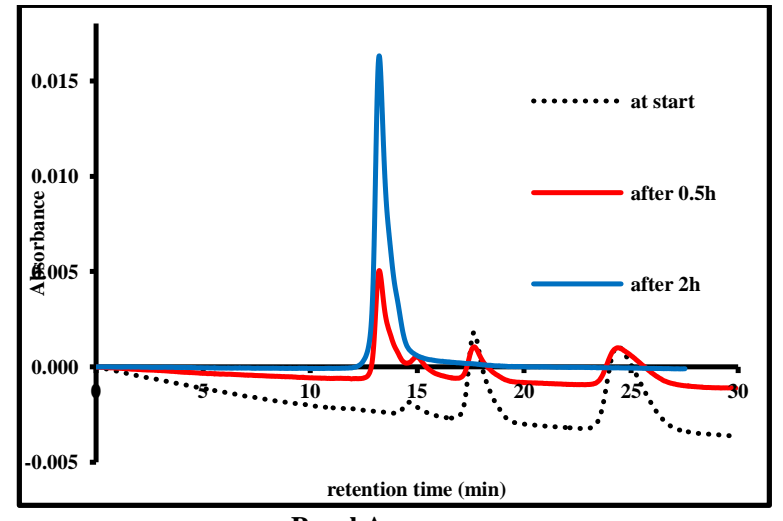

Panel A

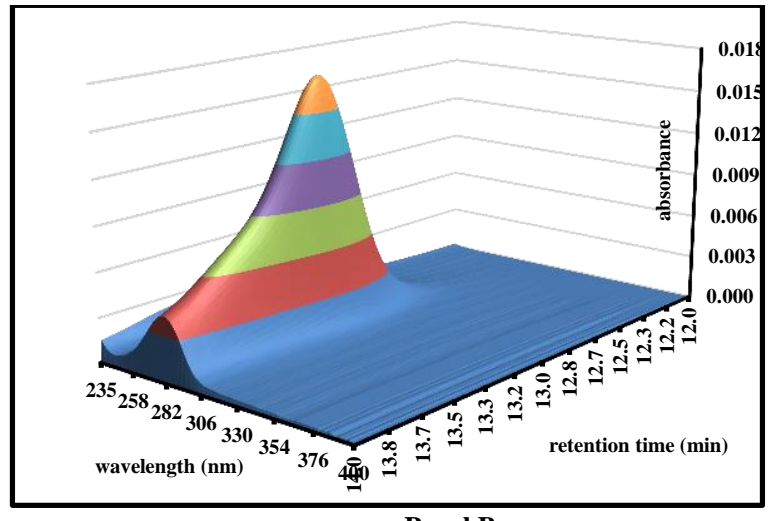

Panel B

Figure 10: SEC profiles at $275 \mathrm{~nm}$ (at the start and after 0.5 and $2 \mathrm{~h}$ reaction) for a reaction mixture containing $0.25 \mathrm{mM}$ dopamine and $0.6 \mathrm{mM}$ serine kept at $37^{\circ} \mathrm{C}$ and the UV-Vis spectra between 235 and $400 \mathrm{~nm}$ of the signal recorded between 12 and 14 minutes retention time (panel B) for the reaction mixture after $2 \mathrm{~h}$ of reaction.

Judging from the rate of disappearance of dopamine, the presence of serine appeared to slow down the decline in dopamine concentration compared to the reaction mixture in the absence of any amino acid. In the SEC profiles shown in Figure 10, panel A, peaks corresponding to reaction intermediates can be observed in the profiles obtained at the start of the reaction and after $0.5 \mathrm{~h}$ of reaction. These intermediates appeared to have disappeared after about $2 \mathrm{~h}$ of reaction. The UV-Vis spectra shown in Figure 10, panel B have a very similar pattern as for the spectra shown in Figure 8, panel B.

Figure 11, panels A and B, illustrate some of the SEC results obtained for a similar reaction mixture as for Figure 8 , but containing $0.6 \mathrm{mM}$ methionine. The mixture was light yellow at the start of the reaction, turning brown after about 30 minutes of reaction and remaining that way for the rest of the observations. About $60 \%$ of the dopamine had reacted away after about 30 minutes of reaction and about $90 \%$ of the dopamine had reacted away after about 150 minutes of reaction. 


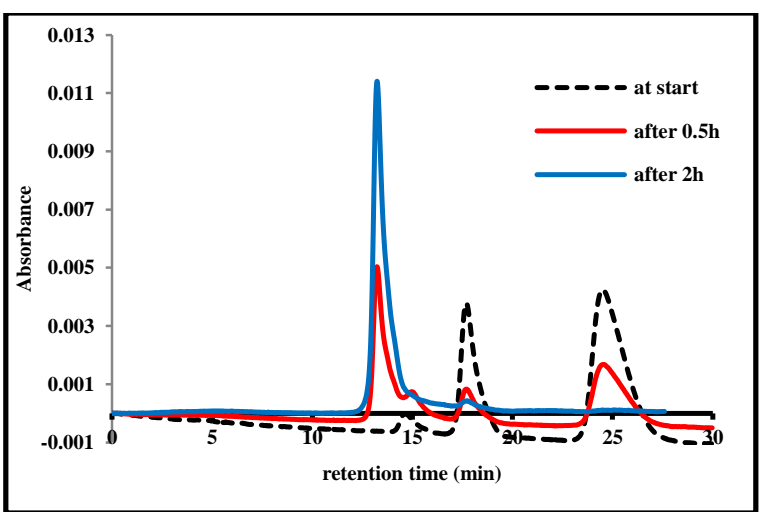

Panel A

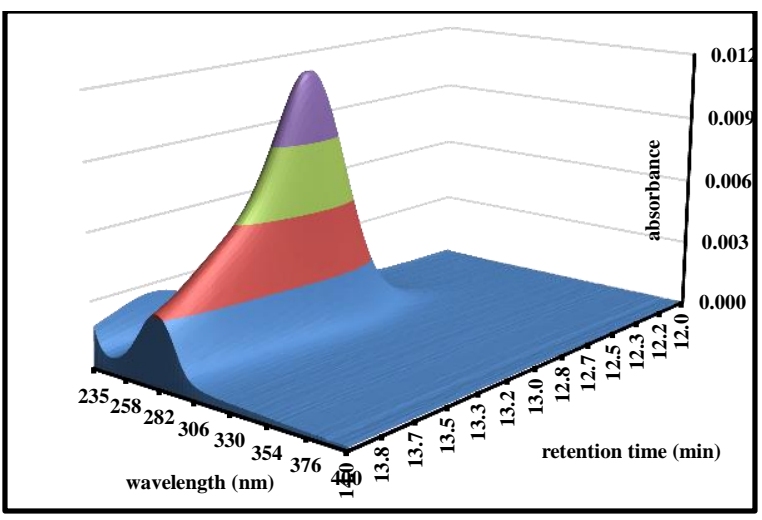

Panel B

Figure 11: SEC profiles at $275 \mathrm{~nm}$ (at the start and after 0.5 and $2 \mathrm{~h}$ reaction) for a reaction mixture containing $0.25 \mathrm{mM}$ dopamine and $0.6 \mathrm{mM}$ methionine kept at $37^{\circ} \mathrm{C}$ and the UV-Vis spectra between 235 and $400 \mathrm{~nm}$ of the signal recorded between 12 and 14 minutes retention time (panel B) for the reaction mixture after $2 \mathrm{~h}$ of reaction.

The results obtained in the presence of methionine as illustrate in Figure 11 are similar to the ones obtained for the reaction in the presence of serine as illustrated in Figure 10. The reaction in the presence of methionine appeared to proceed slower as judged from the disappearance of dopamine.

Figure 12, panels A and B, illustrate some of the SEC results obtained for a similar reaction mixture as for Figure 8 , but containing $0.6 \mathrm{mM}$ tyrosine. The mixture was light yellow at the start of the reaction, turning light-brown after about 30 minutes of reaction and remaining brown after 60 minutes of reaction. About $55 \%$ of the dopamine had reacted away after about 30 minutes of reaction and about $90 \%$ of the dopamine had reacted away after about 90 minutes of reaction.

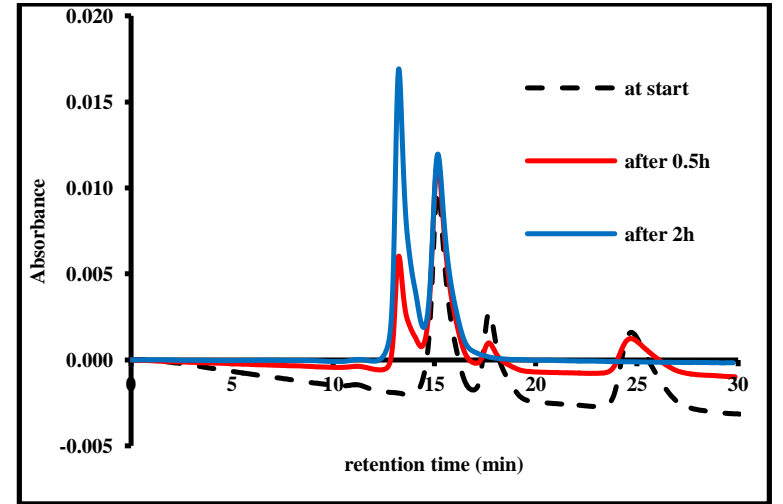

Panel A

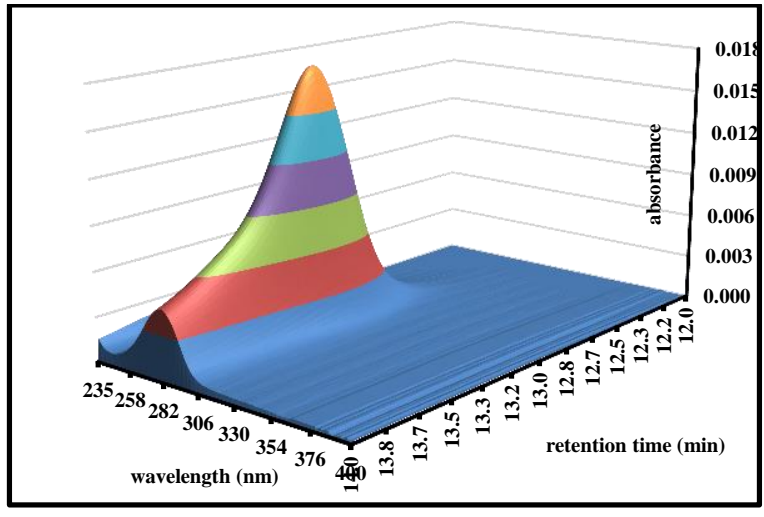

Panel B

Figure 12: SEC profiles at $275 \mathrm{~nm}$ (at the start and after 0.5 and $2 \mathrm{~h}$ reaction) for a reaction mixture containing $0.25 \mathrm{mM}$ dopamine and $0.6 \mathrm{mM}$ tyrosine kept at $37^{\circ} \mathrm{C}$ and the UV-Vis spectra between 235 and $400 \mathrm{~nm}$ of the signal recorded between 12 and 14 minutes retention time (panel B) for the reaction mixture after $2 \mathrm{~h}$ of reaction.

Figure 13, panels A and B, illustrate some of the SEC results obtained for a similar reaction mixture as for Figure 8 , but containing $0.6 \mathrm{mM}$ tyramine. The mixture turned light yellow at the start and was brown in color within 30 minutes of the start of the reaction. After 30 minutes of reaction, about $50 \%$ of the dopamine had reacted away, while almost all of it had disappeared after 90 minutes of reaction. 


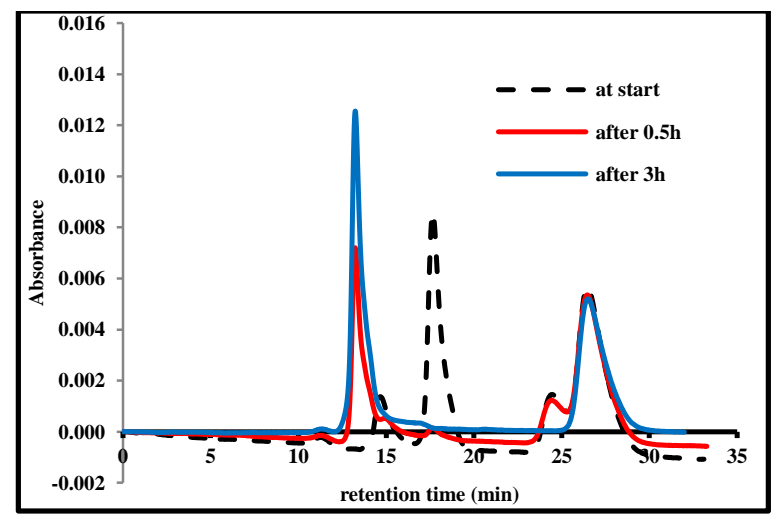

Panel A

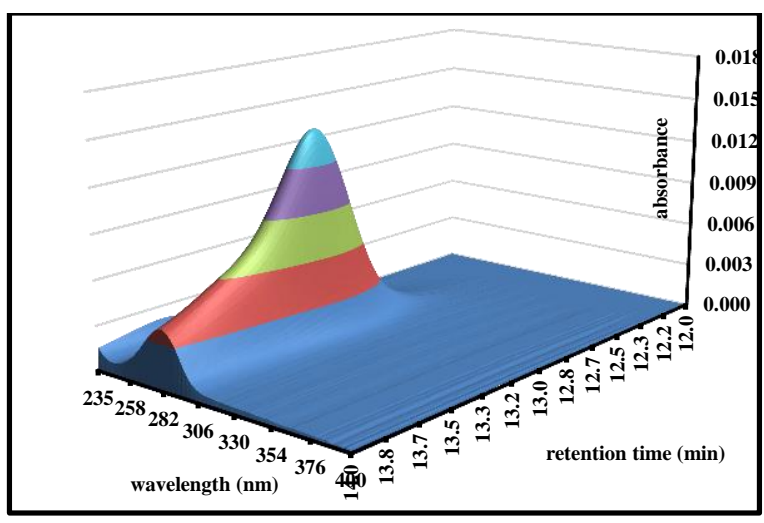

Panel B

Figure 13: SEC profiles at $275 \mathrm{~nm}$ (at the start and after 0.5 and $2 \mathrm{~h}$ reaction) for a reaction mixture containing $0.25 \mathrm{mM}$ dopamine and $0.6 \mathrm{mM}$ tyramine kept at $37^{\circ} \mathrm{C}$ and the UV-Vis spectra between 235 and $400 \mathrm{~nm}$ of the signal recorded between 12 and 14 minutes retention time (panel B) for the reaction mixture after $3 \mathrm{~h}$ of reaction.

The pattern of results obtained for tyrosine and tyramine are similar to the pattern of results obtained for the cases of serine and methionine.

\subsection{SEC studies involving epinephrine or norepinephrine and L-cysteine.}

Reaction mixtures involving norepinephrine or epinephrine $(0.25 \mathrm{mM})$ with L-cysteine $(0.6 \mathrm{mM})$ were set up and sampled for SEC analyses. These mixtures would slowly transition from colorless to light yellow to light brown (norepinephrine) or golden (epinephrine) within the first two hours of the reaction. This coincided with the gradual decrease in concentration of both norepinephrine and epinephrine; with almost all of it reacted away after $2 \mathrm{~h}$ of reaction. Figures 14 and 15 illustrate some of the SEC results obtained from these reactions.

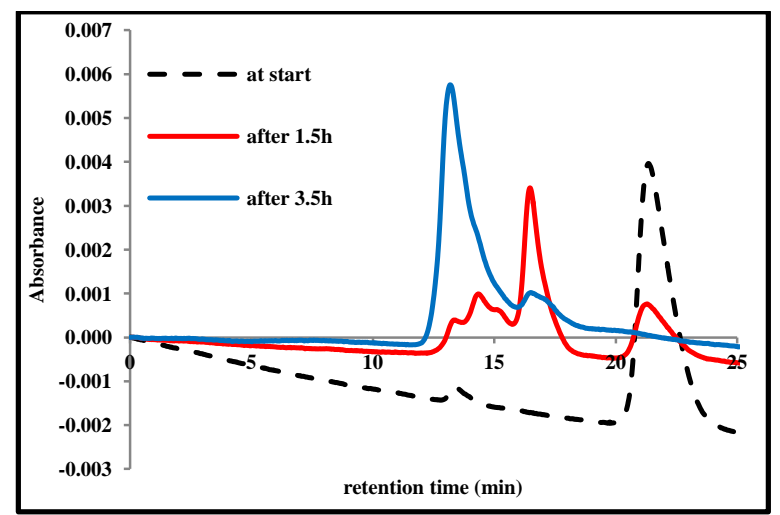

Panel A

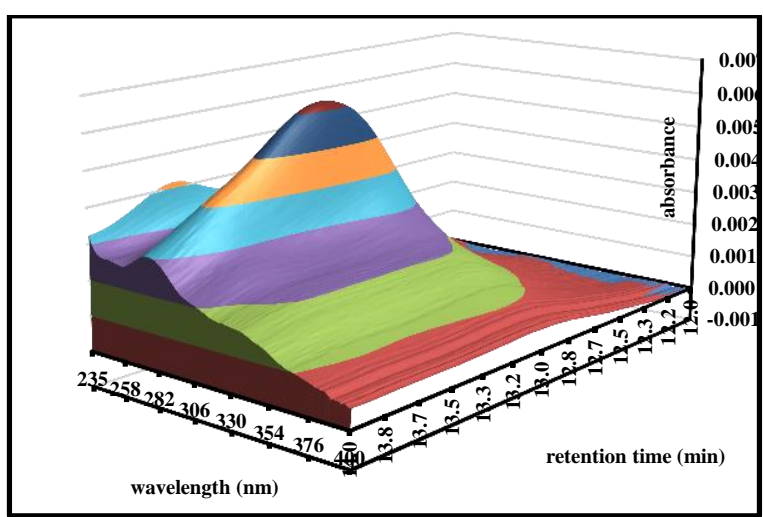

Panel B

Figure 14: SEC profiles at $275 \mathrm{~nm}$ (at the start and after 0.5 and 1.5 or $3.5 \mathrm{~h}$ reaction) for a reaction mixture containing $0.25 \mathrm{mM}$ norepinephrine and $0.6 \mathrm{mM}$ cysteine kept at $37^{\circ} \mathrm{C}$ and the UV-Vis spectra between 235 and $400 \mathrm{~nm}$ of the signal recorded between 12 and 14 minutes retention time (panel B) for the reaction mixture after $3.5 \mathrm{~h}$ of reaction. 


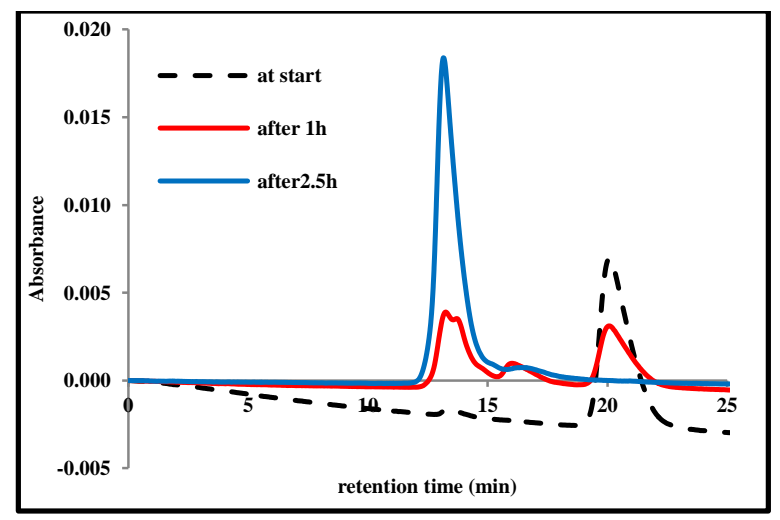

Panel A

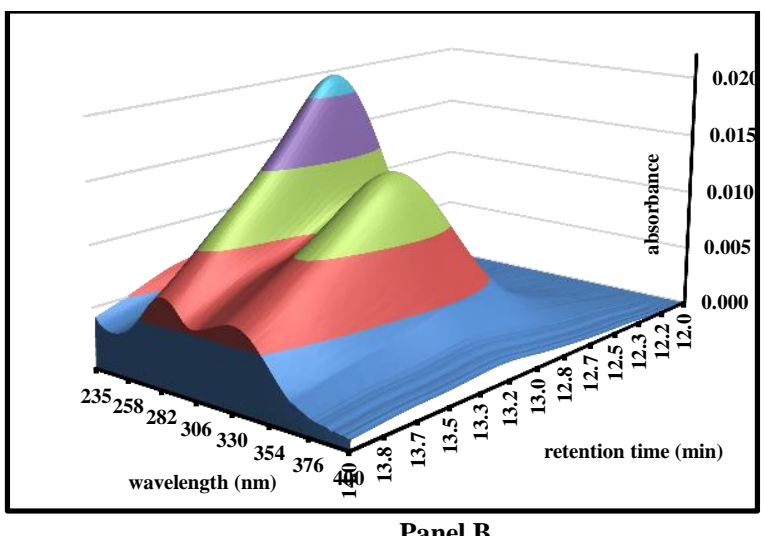

Panel B

Figure 15: SEC profiles at $275 \mathrm{~nm}$ (at the start and after 0.5 and 1.5 or $2.5 \mathrm{~h}$ reaction) for a reaction mixture containing $0.25 \mathrm{mM}$ epinephrine and $0.6 \mathrm{mM}$ cysteine kept at $37^{\circ} \mathrm{C}$ and the UV-Vis spectra between 235 and $400 \mathrm{~nm}$ of the signal recorded between 12 and 14 minutes retention time (panel B) for the reaction mixture after $2.5 \mathrm{~h}$ of reaction.

As observed for dopamine, the auto-oxidation of norepinephrine and epinephrine proceeds slower in the presence of L-cysteine and intermediates of this auto-oxidation process can be observed in the SEC profiles of the reaction mixtures sampled between 0.5 and $1 \mathrm{~h}$ of reaction. Ultimately, the reaction proceeds to completion with all the precursor and intermediates having disappeared and the UV-Vis spectra of the presumed, MN-like material to be very similar to that of the UV-Vis spectra shown in Figure 4, panel E or Figure 5, panel E or the spectra detailed in an earlier report ${ }^{13}$.

\section{Discussion}

This report confirms and expands our observations described elsewhere. ${ }^{13} \mathrm{~L}$-cysteine, in a concentration dependent fashion, delays the onset of color formation during the auto-oxidation reaction of all four catecholamines tested. In the cases of DOPA or dopamine this effect by L-cysteine was very reproducible, while in the cases of norepinephrine or epinephrine there was much greater variability. In addition, the delay in onset of color formation appeared to be much longer in the cases of norepinephrine and epinephrine compared to the cases of DOPA and dopamine. For the other amino acids tested, it was observed that the presence of amino acids slowed the reaction (as judged from the disappearance of the precursor) and the color formation, but did not delay it.

The effect of the amino acids on the kinetics of the auto-oxidation reaction of the catecholamines may reflect itself in the fact that the reaction mixtures were darker in appearance in the presence of amino acids compared to the reaction mixtures in the absence of any added amino acids. In a previous report we have hypothesized that: a) the melanogenesis reaction of catecholamines leads to the synthesis of MN-like material that is built from darklycolored and colorless components, b) that the ratio of these different components would ultimately define the apparent "darkness" of the MN-like material and c) that the kinetics of the melanogenesis reaction may affect the ratio at which these different components; the slower the reaction the darker the material. Thus, as L-cysteine or any other amino acid is capable of slowing down the kinetics of the auto-oxidation reaction, the MN-like materials generated in the presence of amino acids could exhibit a darker appearance. Our current and previous results ${ }^{13}$, together with other reports on the effects of amino acids on the auto-oxidation of catecholic compounds ${ }^{14,16}$, give 
credence to the importance of the presence of amino acids, or any other biomolecules for that matter, on the physicchemical properties of MN-like materials generated during the melanogenesis reaction. Given the importance of pigmentation in biology, any factor that may result in the change of color or a change in intensity of the color is of importance.

It is well-established that the auto-oxidation of catecholamines leads to an endproduct that contains significant amounts of unoxidized precursor units. ${ }^{17,21-23}$ This was observed in our previous and current studies. However, our current studies seem to indicate that the presence of amino acids may generate endproducts that contain intermediates apart from the unoxidized precursor units. In almost all of the reactions studied, these intermediates exhibited a UV-Vis absorbance band or shoulder around 350-370nm (See Figure 6). We hypothesize that, given the effect of amino acids on the kinetics of the melanogenesis reaction, intermediates generated in the auto-oxidation reactions may not react away as quickly as for reactions in the absence of any amino acid. Thus, some of these intermediates may remain "unreacted" within the final MN-like endproduct and provide a second absorbance band apart from the one associated with unreacted precursor. Thus, by studying the melanogenesis reaction in the presence of amino acids, one may get a better glimpse of the intermediates that are formed during this type of reaction. It is worth noting that the results obtained with tyramine hint to the possibility that the effect of primary amines, without the $\alpha$-carbon carboxylic acid, may differ from the effects of their corresponding $\alpha$-amino acids. This would be in line with observations made regarding chlorogenic acid. ${ }^{14,24-25}$ Our preliminary observations regarding tyramine could be expanded to include other biologically relevant primary amines like histamine, tryptamine, $\gamma$-aminobutyric acid, etc.

The effect of L-cysteine on the oxidation process of the catecholamines has been evaluated extensively in the context of its importance in the biosynthesis of pheomelanin ${ }^{18-19,26-31}$ or its importance in the biosynthesis of neuromelanin and the latter's relevance to Parkinson's disease. ${ }^{20,32-40}$ In various reports, Dryhurst et al. studied the oxidation of catecholamines in the presence of L-cysteine. ${ }^{20,32-33,35,41-42}$ These experiments involved electrolytic oxidation reactions at $\mathrm{pH}=7.4$ for 30 to 60 minutes. In the case of dopamine, and in the absence of any L-cysteine, they observed the appearance of DHI and the orange-colored dopaminochrome; two typical intermediates of the oxidation process of dopamine. But a chromatographic analysis revealed that very little dopamine had reacted away under their experimental conditions. Prolonged oxidation conditions did produce a black, insoluble MN-like material. In the presence of 5-fold molar excess L-cysteine, more dopamine reacted away and the reaction mixture contained a complex mixture of compounds; mostly conjugates of L-cysteine and dopamine or its oxidation products. They observed that with increasing concentrations of L-cysteine, the rate of oxidation of dopamine increased and the amount of MN generated decreased. These results are in stark contrast with our previous and current results and we suspect that the major difference in melanogenesis chemistry, auto-oxidation vs. electrolytic chemistry, may be responsible for this contrast. Our chromatographic analytic technique does lack sophisticated resolution possibilities that would allow us for the detection of the various cysteine-based conjugates as reported by Dryhurst et al. However, we can observe the appearance and disappearance of intermediates that, based upon their UV-Vis profile, resemble dopaminochrome or DHI. 
In general, when studying the melanogenesis process, particularly if the color of the final product is of importance, one should consider the effect on this process by any other biomolecule(s) that may be present. Apart from the effect of amino acids reported in this study, polysaccharides ${ }^{43-44}$ or proteins, e.g., PMEL ${ }^{45-46}$, may affect the synthesis and/or disposition of MN-like materials.

\section{References}

1.d'Ischia, M., Melanin-Based Functional Materials. Int J Mol Sci 2018, 19 (1).

2.d'Ischia, M.; Wakamatsu, K.; Cicoira, F.; Di Mauro, E.; Garcia-Borron, J. C.; Commo, S.; Galván, I.; Ghanem, G.; Kenzo, K.; Meredith, P.; Pezzella, A.; Santato, C.; Sarna, T.; Simon, J. D.; Zecca, L.; Zucca, F. A.; Napolitano, A.; Ito, S., Melanins and melanogenesis: from pigment cells to human health and technological applications. Pigm Cell Melanoma Res 2015, 28 (5), 520-544.

3.Solano, F., Melanins: Skin Pigments and Much More:Types, Structural Models, Biological Functions, and Formation Routes. New Journal of Science 2014, 2014, 28.

4.D'Alba, L.; Shawkey, M. D., Melanosomes: Biogenesis, Properties, and Evolution of an Ancient Organelle. Physiol Rev 2019, 99 (1), 1-19.

5.Kohl, F. R.; Grieco, C.; Kohler, B., Ultrafast spectral hole burning reveals the distinct chromophores in eumelanin and their common photoresponse. Chemical Science 2020, 11 (5), 1248-1259.

6.Liebscher, J., Chemistry of Polydopamine - Scope, Variation, and Limitation. European Journal of Organic Chemistry 2019, 2019 (31-32), 4976-4994.

7.Ito, S., Reexamination of the structure of eumelanin. Biochimica et Biophysica Acta (BBA) - General Subjects 1986, 883 (1), 155-161.

8.Simon, J. D.; Peles, D. N., The Red and the Black. Accounts of Chemical Research 2010, 43 (11), 14521460.

9.Cuthill, I. C.; Allen, W. L.; Arbuckle, K.; Caspers, B.; Chaplin, G.; Hauber, M. E.; Hill, G. E.; Jablonski, N. G.; Jiggins, C. D.; Kelber, A.; Mappes, J.; Marshall, J.; Merrill, R.; Osorio, D.; Prum, R.; Roberts, N. W.; Roulin, A.; Rowland, H. M.; Sherratt, T. N.; Skelhorn, J.; Speed, M. P.; Stevens, M.; Stoddard, M. C.; Stuart-Fox, D.; Talas, L.; Tibbetts, E.; Caro, T., The biology of color. Science 2017, 357 (6350). 10.Sudhakar, P.; Latha, P.; Reddy, P. V., Chapter 15 - Plant pigments. In Phenotyping Crop Plants for Physiological and Biochemical Traits, Sudhakar, P.; Latha, P.; Reddy, P. V., Eds. Academic Press: 2016; pp 121-127. 
11.Michaelian, K.; Simeonov, A., Fundamental molecules of life are pigments which arose and coevolved as a response to the thermodynamic imperative of dissipating the prevailing solar spectrum. Biogeosciences 2015, 12 (16), 4913-4937.

12.Michaelian, K., Photochemical Dissipative Structuring of the Fundamental Molecules of Life. Proceedings 2020, 46 (1), 20.

13.Vercruysse, K.; Govan, V., Melanogenesis: A Search for Pheomelanin and Also, What Is Lurking Behind Those Dark Colors? 2019.

14.Iacomino, M.; Weber, F.; Gleichenhagen, M.; Pistorio, V.; Panzella, L.; Pizzo, E.; Schieber, A.; d'Ischia, M.; Napolitano, A., Stable Benzacridine Pigments by Oxidative Coupling of Chlorogenic Acid with Amino Acids and Proteins: Toward Natural Product-Based Green Food Coloring. Journal of agricultural and food chemistry 2017, 65 (31), 6519-6528.

15.Vercruysse, K., Preliminary Observations on the Interactions Between Chlorogenic Acid and Select Amino Acids. ChemRxiv: 2019.

16.Jackson, H.; Kendal, L. P., The oxidation of catechol and homocatechol by tyrosinase in the presence of amino-acids. The Biochemical journal 1949, 44 (4), 477-487.

17.Micillo, R.; Panzella, L.; Koike, K.; Monfrecola, G.; Napolitano, A.; d'Ischia, M., "Fifty Shades" of Black and Red or How Carboxyl Groups Fine Tune Eumelanin and Pheomelanin Properties. Int J Mol Sci 2016, 17 (5).

18.Ito, S.; Wakamatsu, K., Chemistry of mixed melanogenesis--pivotal roles of dopaquinone. Photochem Photobiol 2008, 84 (3), 582-92.

19.Agrup, G.; Hansson, C.; Rorsman, H.; Rosengren, E., The effect of cysteine on oxidation of tyrosine, dopa, and cysteinyldopas. Arch Dermatol Res 1982, 272 (1-2), 103-15.

20.Shen, X.-M.; Dryhurst, G., Oxidation Chemistry of (-)-Norepinephrine in the Presence of I-Cysteine. Journal of Medicinal Chemistry 1996, 39 (10), 2018-2029.

21.Ambrico, M.; Ambrico, P. F.; Cardone, A.; Della Vecchia, N. F.; Ligonzo, T.; Cicco, S. R.; Talamo, M. M.; Napolitano, A.; Augelli, V.; Farinola, G. M.; d'Ischia, M., Engineering polydopamine films with tailored behaviour for next-generation eumelanin-related hybrid devices. Journal of Materials Chemistry C 2013, 1 (5), 1018-1028.

22.Ball, V.; Gracio, J.; Vila, M.; Singh, M. K.; Metz-Boutigue, M. H.; Michel, M.; Bour, J.; Toniazzo, V.; Ruch, D.; Buehler, M. J., Comparison of synthetic dopamine-eumelanin formed in the presence of oxygen and Cu2+ cations as oxidants. Langmuir 2013, 29 (41), 12754-61. 
23.Carstam, R.; Brinck, C.; Hindemith-Augustsson, A.; Rorsman, H.; Rosengren, E., The neuromelanin of the human substantia nigra. Biochim Biophys Acta 1991, 1097 (2), 152-60.

24.Prigent, S. V.; Voragen, A. G.; Li, F.; Visser, A. J.; van Koningsveld, G. A.; Gruppen, H., Covalent interactions between amino acid side chains and oxidation products of caffeoylquinic acid (chlorogenic acid). Journal of the Science of Food and Agriculture 2008, 88 (10), 1748-1754.

25.Schilling, S.; Sigolotto, C. I.; Carle, R.; Schieber, A., Characterization of covalent addition products of chlorogenic acid quinone with amino acid derivatives in model systems and apple juice by highperformance liquid chromatography/electrospray ionization tandem mass spectrometry. Rapid Commun Mass Spectrom 2008, 22 (4), 441-8.

26.Ito, S.; Prota, G., A facile one-step synthesis of cysteinyldopas using mushroom tyrosinase. Experientia 1977, 33 (8), 1118-9.

27.Ito, S.; Fujita, K., Formation of cysteine conjugates from dihydroxyphenylalanine and its S-cysteinyl derivatives by peroxidase-catalyzed oxidation. Biochim Biophys Acta 1981, 672 (2), 151-7.

28.Ito, S.; Fujita, K., Conjugation of dopa and 5-S-cysteinyldopa with cysteine mediated by superoxide radical. Biochem Pharmacol 1982, 31 (18), 2887-9.

29.Agrup, G.; Edholm, L. E.; Rorsman, H.; Rosengren, E., Diastereomers of 5-S-cysteinyldopa. Acta Derm Venereol 1983, 63 (1), 59-61.

30.Ito, S.; Fujita, K., Oxygen-dependent conjugation of dopa with cysteine catalysed by iron-EDTA complex. Biochem Pharmacol 1984, 33 (14), 2193-7.

31.Ito, S., Optimization of conditions for preparing synthetic pheomelanin. Pigment Cell Res 1989, 2 (1), 53-6.

32.Zhang, F.; Dryhurst, G., Effects of L-cysteine on the oxidation chemistry of dopamine: new reaction pathways of potential relevance to idiopathic Parkinson's disease. J Med Chem 1994, 37 (8), 1084-98.

33.Shen, X. M.; Dryhurst, G., Further insights into the influence of L-cysteine on the oxidation chemistry of dopamine: reaction pathways of potential relevance to Parkinson's disease. Chemical research in toxicology 1996, 9 (4), 751-63.

34.Shen, X. M.; Zhang, F.; Dryhurst, G., Oxidation of dopamine in the presence of cysteine: characterization of new toxic products. Chemical research in toxicology 1997, 10 (2), 147-55.

35.Shen, X.-M.; Dryhurst, G., Further Insights into the Oxidation Chemistry of Norepinephrine and Epinephrine in the Presence of Cysteine. Bioorganic Chemistry 1997, 25 (2), 130-153. 
36.Shen, X. M.; Dryhurst, G., Iron- and manganese-catalyzed autoxidation of dopamine in the presence of L-cysteine: possible insights into iron- and manganese-mediated dopaminergic neurotoxicity. Chemical research in toxicology 1998, 11 (7), 824-37.

37.Xin, W.; Shen, X. M.; Li, H.; Dryhurst, G., Oxidative metabolites of 5-S-cysteinylnorepinephrine are irreversible inhibitors of mitochondrial complex I and the alpha-ketoglutarate dehydrogenase and pyruvate dehydrogenase complexes: possible implications for neurodegenerative brain disorders. Chemical research in toxicology 2000, 13 (8), 749-60.

38.Li, H.; Dryhurst, G., Oxidative metabolites of 5-S-cysteinyldopamine inhibit the pyruvate dehydrogenase complex. J Neural Transm (Vienna) 2001, 108 (12), 1363-74.

39.Manini, P.; Panzella, L.; Napolitano, A.; d’Ischia, M., Oxidation Chemistry of Norepinephrine: Partitioning of the O-Quinone between Competing Cyclization and Chain Breakdown Pathways and Their Roles in Melanin Formation. Chemical research in toxicology 2007, 20 (10), 1549-1555.

40.Wakamatsu, K.; Tabuchi, K.; Ojika, M.; Zucca, F. A.; Zecca, L.; Ito, S., Norepinephrine and its metabolites are involved in the synthesis of neuromelanin derived from the locus coeruleus. $J$ Neurochem 2015, 135 (4), 768-76.

41.Shen, X.-M.; Dryhurst, G., Influence ofL-Cysteine on the Oxidation Chemistry of (-)-Epinephrine: Formation of Cysteinyl Conjugates and Novel Dihydrobenzothiazines. Bioorganic Chemistry 1996, 24 (4), 340-357.

42.Shen, X.-M.; Xia, B.; Wrona, M. Z.; Dryhurst, G., Synthesis, Redox Properties, in Vivo Formation, and Neurobehavioral Effects of N-Acetylcysteinyl Conjugates of Dopamine: Possible Metabolites of Relevance to Parkinson's Disease. Chemical research in toxicology 1996, 9 (7), 1117-1126.

43.Vercruysse, K.; Clark, A.; Alatas, N.; Brooks, D.; Hamza, N.; Whalen, M., Polysaccharide-mediated synthesis of melanins from serotonin and other 5-hydroxy indoles. Future Science OA 2018, FSO280.

44.Vercruysse, K. P.; Clark, A. M.; Bello, P. A. F.; Alhumaidi, M., Using size exclusion chromatography to monitor the synthesis of melanins from catecholamines. J Chromatogr B 2017, 1061-1062, 11-16.

45.Bissig, C.; Rochin, L.; van Niel, G., PMEL Amyloid Fibril Formation: The Bright Steps of Pigmentation. Int J Mol Sci 2016, 17 (9).

46.Chakraborty, A. K.; Platt, J. T.; Kim, K. K.; Kwon, B. S.; Bennett, D. C.; Pawelek, J. M., Polymerization of 5,6-dihydroxyindole-2-carboxylic acid to melanin by the pmel 17/silver locus protein. Eur J Biochem 1996, $236(1), 180-8$. 